\title{
Locally finitely presented categories with no flat objects
}

\author{
Sergio Estrada \\ sestrada@um.es \\ Departamento de Matemática Aplicada \\ Universidad de Murcia \\ 30100 Murcia \\ SPAIN \\ Manuel Saorín \\ msaorinc@um.es \\ Departamento de Matemáticas \\ Universidad de Murcia \\ 30100 Murcia \\ SPAIN *
}

\begin{abstract}
If $X$ is a quasi-compact and quasi-separated scheme, the category $Q \operatorname{coh}(X)$ of quasi-coherent sheaves on $X$ is locally finitely presented. Therefore categorical flat quasi-coherent sheaves in the sense of [30] naturally arise. But there is also the standard definition of flatness in $Q \operatorname{coh}(X)$ from the stalks. So it makes sense to wonder the relationship (if any) between these two notions. In this paper we show that there are plenty of locally finitely presented categories having no other categorical flats than the zero object. As particular instance, we show that $\left.Q \operatorname{coh}\left(\mathbf{P}^{n}(R)\right)\right)$ has no other categorical flat objects than zero, where $R$ is any commutative ring.
\end{abstract}

2010 Mathematics Subject Classification. 18E15,18C35,18F20,18A40,16G20

Key words and phrases: locally finitely presented category, Grothendieck category, flat object, quasi-coherent sheaf, projective scheme, quiver representation .

\section{Introduction}

It is widely known that locally finitely presented additive categories are the natural framework for considering a theory of purity (see [6]). We recall that a locally finitely presented additive category $\mathcal{G}$ is an additive category with direct limits such that every object is a direct limit of finitely presented objects, and the class of finitely presented objects is skeletally small. And a sequence $0 \rightarrow L \stackrel{f}{\rightarrow} M \stackrel{g}{\rightarrow} N \rightarrow 0$ in $\mathcal{G}$ is pure if

$$
0 \rightarrow \operatorname{Hom}(T, L) \rightarrow \operatorname{Hom}(T, M) \rightarrow \operatorname{Hom}(T, N) \rightarrow 0
$$

*The authors have been partially supported by the DGI MTM2010-20940-C02-02 and by the Fundación Seneca 04555/GERM/06. 
is exact, for each finitely presented $T$ of $\mathcal{G}$ (in this case $g$ is said to be a pure epimorphism). Therefore locally finitely presented additive categories come equipped with a canonical notion of flat object (in the sense of Stenström [30]) which is defined in terms of pure epimorphisms (this definition is equivalent to that of [6, Section 1.3]). Namely, $F$ is flat if every epimorphism $M \rightarrow N$ is a pure epimorphism.

Moreover, if we assume that $\mathcal{G}$ has enough projectives then it is not difficult to check that flat objects are precisely the objects satisfying a Govorov-Lazard like theorem. The Govorov-Lazard Theorem says that the closure under direct limits of the class of finitely generated projective objects is equal to the class of flat objects (see [12] and [18. Théorème 1.2]). We point out that a flat and finitely presented object must be necessarily projective. Let us denote by $\mathcal{F l a t}$ the class of all flat objects in a locally finitely presented category. Despite of the Govorov-Lazard theorem, this class has homological significance as well. In 7, 28, is proved, as a consequence, that $\mathcal{F}$ lat provides with minimal flat resolutions that are unique up to homotopy, so they can be used to compute right derived functors of the Hom functor. Also the existence of such minimal approximations with respect to Flat can be used to infer the existence of pure-injective envelopes in a locally finitely presented category (see [15]).

However, when working with the category $Q \operatorname{coh}(X)$ of quasi-coherent sheaves on a scheme, flatness of a sheaf is defined in terms of the flatness of its stalks. And, in general, this geometrical notion of flatness differs from Stenström's in the non-affine case. For instance, if $X=\mathbf{P}^{1}(R)$ (with $R$ any commutative ring) the line bundle $\mathcal{O}(n)$ are finitely presented and flat, but not projective. In addition, most of the schemes $X$ that occur in practice in algebraic geometry (like quasi-compact and quasi-separated schemes) are such that $Q \operatorname{coh}(X)$ is locally finitely presented (see [13, I.6.9.12]), so one also might consider the class $\mathcal{F l a t}$ on $Q \operatorname{coh}(X)$ as a natural choice. We point out that the existence of unique up-to-homotopy geometrical flat resolutions is guaranteed by [9, Section 5]. So a natural question arises: is there any relation between these two notions of flatness in $Q \operatorname{coh}(X)$ ?. Whereas geometrical flatness is well-known and widely studied in Algebraic Geometry, this seems not to be the case of the class Flat in $Q \operatorname{coh}(X)$ ( $X$ quasi-compact and quasi-separated), although in view of the previous comments it turns out to be a canonical choice on each locally finitely presented additive category. We note that Crivei, Prest and Torrecillas have recently considered this dichotomy of the two notions of flatness in categories of sheaves of modules in [7, Section 3].

Thus we devote this paper to study the class $\mathcal{F l a t}$ of flat objects in locally finitely presented Grothendieck categories. To be more precise, we study the lack of flat objects (apart from the zero object) in such categories. We find sufficient conditions on a locally finitely presented Grothendieck category to ensure that it contains no nonzero flats. They allow to construct a plethora of examples of locally finitely presented Grothendieck categories having no other flats than the zero object, showing that this behavior is not pathological at all in locally finitely presented categories. To illustrate this we give examples from commutative algebra, algebraic geometry and representation theory.

Now we will formulate a more precise statements of the resuls of the paper. Our setup on the locally finitely presented category $\mathcal{G}$ is based upon a technical assumption on the generators of $\mathcal{G}$ as well as in considering a hereditary torsion class of finite type on $\mathcal{G}$ (proposition 2.5). The first set of applications (theorem 3.2) deals with the quotient category of graded modules modulo the virtually finitely graded ones (see Section 3 for unexplained notation and terminology):

Theorem 1.1. Let $A=\oplus_{n \geq 0} A_{n}$ be a positively graded ring with the property that $A_{\geq 1}$ is finitely presented and $A_{\geq n}$ is finitely generated, as left ideals, for all $n>>0$. Let $\mathcal{T}$ be the class of virtually finitely graded left $A$-modules and $t$ the associated torsion radical. The category $A-G r / \mathcal{T}$ is locally finitely presented and, in case $\operatorname{Ext}_{A}^{1}\left(A_{0}, A / t(A)\right)=0$, it has no nonzero flat objects.

The second one, theorem 4.4 relies on the category of quasi-coherent sheaves on a projective scheme:

Theorem 1.2. Let $A=\oplus_{n \geq 0} A_{n}$ be a positively graded commutative ring which is finitely generated by $A_{1}$ as an $A_{0}$-algebra, let $I$ be the (graded) ideal of $A$ consisting of those $a \in A$ such that $A_{1}^{n} a=0$, for some $n=n(a) \in \mathbb{N}$, and let $X=\operatorname{Proj}(A)$ be the associated projective scheme. If the two following conditions hold, then $Q \operatorname{coh}(X)$ has no nonzero categorical flat object: 
1. The ideal $A_{\geq 1}$ is finitely presented.

2. $\operatorname{Ext}_{A}^{1}\left(A_{0}, A / I\right)=0$

Conditions (1) and (2) of the previous theorem may look rather technical at first glance. But it is shown in corollary 4.5 that it covers many natural projective schemes, like $\operatorname{Proj}(A)$ whenever $A$ is integrally closed or Cohen-Macaulay positively graded commutative Noetherian domain. It also includes the case $X=\mathbf{P}^{n}(R)$, the projective $n$-space over a commutative ring $R$ (corollary 4.6). The last of the application (theorem 5.6) goes back to quotient categories of categories of representations of a quiver with relations $(Q, \rho)$ over an arbitrary commutative ring:

Theorem 1.3. Let $A$ be an algebra with enough idempotents locally finitely presented by the quiver with relations $(Q, \rho)$, where $Q$ is connected, locally finite and has no oriented cycles. Let $A=B \oplus J$ its canonical Wedderburn-Malcev type decomposition and let $\mathcal{T}$ be the class of torsion A-modules, whose torsion radical is denoted by $t$. The following assertions hold:

1. If $\rho$ consists of homogeneous relations, $t(A)=0$ and, for each $i \in Q_{0}$, there are only finitely many vertices $j$ such that $i \preceq j$, then $\mathcal{T}$ is a locally finitely presented Grothendieck category with no nonzero flat objects.

2. If $Q$ is downward directed and narrow and $\operatorname{Ext}_{A}^{1}\left(B e_{i}, \frac{A e_{j}}{t(A) e_{j}}\right)=0$, for all $i, j \in Q_{0}$, then $\mathcal{G} / \mathcal{T}$ is a locally finitely presented Grothendieck category with no nonzero flat objects.

The previous result provides an ample range of examples of categories with no nonzero flat objects. For instance, it applies to the category of comodules of a wide class of coalgebras as it is shown in corollary [5.7 (cf. [8, Example 2.11]), and also to certain quotient categories built up around a Cohen-Macaulay or an integrally closed Noetherian integral domain (see corollary 5.8).

\section{Construction of locally finitely presented categories without flat objects}

All throughout these notes, the letter $\mathcal{G}$ denotes a Grothendieck category.

Definition 1. Let I be a downward directed preordered set which has not a minimum. A set of generators $\mathcal{S}$ of $\mathcal{G}$ will be called densely $I$-stratified when it can be expressed as a union $\mathcal{S}=\bigcup_{i \in I} \mathcal{S}_{i}$ satisfying the following properties:

1. $\mathcal{S}_{i} \neq \emptyset$, for each $i \in I$

2. For each $i \in I$ and each $X \in \mathcal{S}_{i}$, there are only finitely many indices $j<i$ with the property that $\operatorname{Hom}_{\mathcal{G}}(X, ?)_{\mid \mathcal{S}_{j}} \neq 0$

3. For all $i, j \in I$ such that $j<i$ and each $X \in \mathcal{S}_{i}$, there is an epimorphism $X^{\prime} \rightarrow X$ in $\mathcal{G}$, where $X^{\prime}$ is a coproduct of objects in $\bigcup_{k \leq j} \mathcal{S}_{j}$.

We will simply say that $\mathcal{S}$ is densely stratified when it is densely I-stratified, for some downward directed preordered set I as above.

Definition 2. An object $X$ of $\mathcal{G}$ is called finitely presented when the functor $\operatorname{Hom}_{\mathcal{G}}(X, ?): \mathcal{G} \longrightarrow A b$ preserves direct limits. The category $\mathcal{G}$ is called locally finitely presented when it has a set of finitely presented generators and each object of $\mathcal{G}$ is a direct limit of finitely presented objects.

(See [30] for the next terms). If $\mathcal{G}$ is locally finitely presented, then an epimorphism $M \stackrel{p}{\rightarrow} N$ in $\mathcal{G}$ is called a pure epimorphism when the induced map $\operatorname{Hom}_{\mathcal{G}}(X, p): \operatorname{Hom}_{\mathcal{G}}(X, M) \longrightarrow \operatorname{Hom}_{\mathcal{G}}(X, N)$ is surjective, for each finitely presented object $X$.

An object $F$ of $\mathcal{G}$ will be called flat when every epimorphism $M \rightarrow F$ is pure. 
Remark 2.1. If $\mathcal{G}$ is locally finitely presented, then the class $f p(\mathcal{G})$ of its finitely presented objects is skeletally small, i.e., the isoclasses of its objects form a set (see [1, Remark 1.9]).

Proposition 2.2. Let $\mathcal{G}$ be locally finitely presented and suppose that it has a densely stratified set $\mathcal{S}$ of finitely presented generators. Then the only flat object in $\mathcal{G}$ is the zero object.

Proof. Let $\mathcal{S}$ be densely $I$-stratified, where $I$ is a downward directed set without minimum. Suppose that $F$ is a nonzero flat object in $\mathcal{G}$. We then have an epimorphism $p: \coprod_{j \in I} X_{j} \rightarrow F$, where $X_{j}$ is a coproduct of objects in $\mathcal{S}_{j}$, for each $j \in I$. This epimorphism is pure since $F$ is flat.

Let now $f: M \longrightarrow F$ be any nonzero morphism, where $M$ is a finitely presented object in $\mathcal{G}$. There is a finite subset $H \subset I$ together with an epimorphism $q: \coprod_{i \in H} Y_{i} \rightarrow M$, where $Y_{i}$ is a finite coproduct of objects of $\mathcal{S}_{i}$, for each $i \in H$. By condition 2 in definition [1, for each $i \in H$, we know that the set

$$
H_{i}^{\prime}=\left\{j \in I: j<i \text { and } \operatorname{Hom}_{\mathcal{G}}\left(Y_{i}, ?\right)_{\mid \mathcal{S}_{j}} \neq 0\right\}
$$

is finite. Now the downward directed condition of $I$ and the nonexistence of minimum imply that, for each $j \in I$ we can find an element $k(j) \in I$ such that $k(j)<k$, for all $k \in\left(\bigcup_{i \in H} H_{i}^{\prime}\right) \cup\{j\}$. Now condition 3 of definition 1 implies the existence of an epimorphism $X_{j}^{\prime} \rightarrow X_{j}$, where $X_{j}^{\prime}$ is a coproduct of objects in $\bigcup_{k \leq k(j)} \mathcal{S}_{k}$, for each $j \in I$. It follows that we have an epimorphism $v: \coprod_{j \in I} X_{j}^{\prime} \rightarrow \coprod_{j \in I} X_{j}$ and, hence, another one $p \circ v: \coprod_{j \in I} X_{j}^{\prime} \rightarrow F$.

The epimorphism $p \circ v$ is pure since $F$ is flat and, as consequence, there is a morphism $g: M \longrightarrow$ $\coprod_{j \in I} X_{j}^{\prime}$ such that $f=p \circ v \circ g$. But then we have the morphism $g \circ q: \coprod_{i \in H} Y_{i} \longrightarrow \coprod_{j \in I} X_{j}^{\prime}$. This morphism is necessarily zero since each $X_{j}^{\prime}$ is a coproduct of objects in $\bigcup_{k \leq k(j)} \mathcal{S}_{k}$, for all $j \in I$, and $\operatorname{Hom}_{\mathcal{G}}\left(Y_{i}, ?\right)_{\mid \mathcal{S}_{k}}=0$ for all such $k$. Therefore $g \circ q=0$, which implies that $g=0$, and so $f=0$, since $q$ is an epimorphism. We then get a contradiction with the fact that $f \neq 0$.

We now recall certain facts about localization of Grothendieck categories (see [10, [31]). If $\mathcal{T}$ is a hereditary torsion class of $\mathcal{G}$ (i.e. $\mathcal{T}$ is closed under taking subobjects, quotients, extensions and coproducts in $\mathcal{G}$ ) then the inclusion functor $i: \mathcal{T} \hookrightarrow \mathcal{G}$ has a right adjoint $t: \mathcal{G} \longrightarrow \mathcal{T}$, and hence the unit $1_{\mathcal{T}} \longrightarrow t \circ i$ of this adjunction is an isomorphism. It follows that $(i \circ t)^{2}$ is naturally isomorphic to $i \circ t$. For simplification purposes, we will abuse of notation and denote also by $t$ the composition $i$ o $t$. Then we interpret $t$ as an idempotent functor $t: \mathcal{G} \longrightarrow \mathcal{G}$, actually a subfunctor of the identity, called the torsion radical associated to $\mathcal{T}$. For each object $M$ of $\mathcal{G}$, the subobject $t(M)$ is the largest one which is in $\mathcal{T}$. Then $M$ fits into an exact sequence

$$
0 \rightarrow t(M) \stackrel{\text { incl }}{\longrightarrow} M \longrightarrow M / t(M) \rightarrow 0,
$$

which is natural on $M$. We will denote by $\mathcal{T}^{\perp}$ the class of objects $Y$ such that $\operatorname{Hom}_{\mathcal{G}}(T, Y)=0$, for all $T \in \mathcal{T}$. Then $M / t(M)$ is in $\mathcal{T}^{\perp}$.

There is a Grothendieck category $\mathcal{G} / \mathcal{T}$, called the quotient category of $\mathcal{G}$ modulo $\mathcal{T}$, together with functor $q: \mathcal{G} \longrightarrow \mathcal{G} / \mathcal{T}$, called the quotient functor, which is universal with respect to each one of the following properties:

1. It is an additive functor, with domain $\mathcal{G}$ and codomain another Grothendieck category, which vanishes on all objects of $\mathcal{T}$.

2. It is an additive functor, with domain $\mathcal{G}$ and codomain another Grothendieck category, which takes each morphism with kernel and cokernel in $\mathcal{T}$ to an isomorphism.

In such case, it is well-known that $q$ is exact and has a right adjoint $\iota: \mathcal{G} / \mathcal{T} \longrightarrow \mathcal{G}$, usually called the section functor, which is fully faithful (equivalently, the counit of the adjunction, $\epsilon: q \circ \iota \longrightarrow 1_{\mathcal{G} / \mathcal{T}}$, is an isomorphism). Moreover, if $\mu: 1_{\mathcal{G}} \longrightarrow \iota \circ q$ is the unit of this adjunction, then each object $M \in \mathcal{G}$ fits into an exact sequence

$$
0 \rightarrow t(M) \stackrel{\text { incl }}{\longrightarrow} M \stackrel{\mu_{M}}{\longrightarrow}(\iota \circ q)(M) \longrightarrow T_{M} \rightarrow 0
$$


where $T_{M} \in \mathcal{T}$.

From the last exact sequence it easily follows that $M$ is in the essential image of $\iota$ if, and only if, $\operatorname{Hom}_{\mathcal{G}}(T, M)=0=\operatorname{Ext}_{\mathcal{G}}^{1}(T, M)$ for all $T \in \mathcal{T}$. In the sequel, we shall freely identify $\mathcal{G} / \mathcal{T}$ with $\operatorname{Im}(\iota)$ whenever necessary, looking at $\mathcal{G} / \mathcal{T}$ in that way as a full subcategory of $\mathcal{G}$.

The following result will be very useful later on:

Proposition 2.3. Let $j: \mathcal{G}^{\prime} \longrightarrow \mathcal{G}$ be an additive functor between Grothendieck categories. Suppose that it has left adjoint $p: \mathcal{G} \longrightarrow \mathcal{G}^{\prime}$ which is exact and such that the counit $\epsilon: p \circ j \longrightarrow 1_{\mathcal{G}^{\prime}}$ is an isomorphism. Then the following assertions hold:

1. $\mathcal{T}:=\operatorname{Ker}(p)$ is a hereditary torsion class in $\mathcal{G}$

2. The induced additive functor $\mathcal{G} / \mathcal{T} \longrightarrow \mathcal{G}^{\prime}$ is an equivalence of categories.

Proof. 1) The functor $p$ preserves coproducts since it is left adjoint. It follows that $\mathcal{T}$ is closed under taking coproducts. On the other hand, the exactness of $p$ implies that, given an exact sequence $0 \rightarrow$ $L \longrightarrow M \longrightarrow N \rightarrow 0$ in $\mathcal{G}$, one has that $M \in \mathcal{T}$ if and only if $L$ and $N$ are in $\mathcal{T}$. Therefore $\mathcal{T}$ is a hereditary torsion class in $\mathcal{G}$.

2) By usual properties of adjunctions, we know that $j$ is fully faithful and, hence, we can identify $\mathcal{G}^{\prime}$ with its essential image by $j$ and view it as a full subcategory of $\mathcal{G}$.

The universal property of the quotient category gives an induced functor $\psi: \mathcal{G} / \mathcal{T} \longrightarrow \mathcal{G}^{\prime}$ such that $\psi \circ q \cong p$, where $q: \mathcal{G} \longrightarrow \mathcal{G} / \mathcal{T}$. Actually $\psi$ acts on objects by taking $q(M) \rightsquigarrow p(M)$, for each $M \in \mathcal{G}$. It immediately follows that $\psi$ is dense (=representative), because, due to the fact that $\epsilon$ is an isomorphism, the functor $p$ is dense. Let us note now that if we identify $\mathcal{G} / \mathcal{T}$ with its image by the section functor $\iota: \mathcal{G} / \mathcal{T} \longrightarrow \mathcal{G}$, then the functor $\psi$ is just the restriction of $p$ to $\operatorname{Im}(\iota)$. Indeed, if $Y \in \operatorname{Im}(\iota)$ then we have $q(Y)=Y$ and so $\psi(Y)=p(Y)$. As a consequence, we know that the restriction of $p$ to $\operatorname{Im}(\iota)$ is a dense functor.

We shall prove that $\operatorname{Im}(\iota) \subseteq \mathcal{G}^{\prime}=\operatorname{Im}(j)$, and from this and the preceding paragraph assertion 2 will follow since the restriction of $p$ to $\operatorname{Im}(j)$ is the identity (recall that $\epsilon$ is an isomorphism!). To see that $\operatorname{Im}(\iota) \subseteq \mathcal{G}^{\prime}$, we use the adjunction equations. If $\eta: 1_{\mathcal{G}} \longrightarrow j \circ p$ is the unit of the adjunction $p \vdash j$, then $p\left(\eta_{M}\right): p(M) \longrightarrow(p \circ j \circ p)(M)$ is an isomorphism, for each $M \in \mathcal{G}$. Due to the exactness of $p$ this is equivalent to saying that $\operatorname{Ker}\left(\eta_{M}\right)$ and $\operatorname{Im}\left(\eta_{M}\right)$ are objects of $\mathcal{T}$. But if $M \in \operatorname{Im}(\iota)$ then $\operatorname{Hom}_{\mathcal{G}}(T, M)=$ $0=\operatorname{Ext}_{\mathcal{G}}^{1}(T, M)$, for all $T \in \mathcal{T}$, from which it follows $\operatorname{Ker}\left(\eta_{M}\right)=0$ and, hence, that $\eta_{M}$ is a section $(=$ split monomorphism $)$ in $\mathcal{G}$. But $\operatorname{Hom}_{\mathcal{G}}(T,(j \circ p)(M)) \cong \operatorname{Hom}_{\mathcal{G}^{\prime}}(p(T), p(M))=\operatorname{Hom}_{\mathcal{G}^{\prime}}(0, p(M))=0$, for all $T \in \mathcal{T}$. It follows that any morphism $\operatorname{Coker}\left(\eta_{M}\right) \longrightarrow(j \circ p)(M)$ is the zero morphism, and therefore $\eta_{M}$ is an isomorphism. It follows that $M \in \operatorname{Im}(j)=\mathcal{G}^{\prime}$, and hence $\operatorname{Im}(\iota) \subseteq \mathcal{G}^{\prime}$.

Recall that if $\mathcal{G}$ is locally finitely presented, then the hereditary torsion class $\mathcal{T}$ is called of finite type when the section functor $\iota: \mathcal{G} / \mathcal{T} \longrightarrow \mathcal{G}$ preserves direct limits or, equivalently, when $\operatorname{Im}(\iota)$ is closed under taking direct limits in $\mathcal{G}$.

The following result seems to be folklore and it can be derived from [27]. (see also [16, Proposition A5] for a particular case). We include here a short proof for the sake of completeness.

Proposition 2.4. Let $\mathcal{G}$ be a locally finitely presented Grothendieck category, let $\mathcal{S}$ be a set of finitely presented generators and let $\mathcal{T}$ be a hereditary torsion class of finite type. Then $\mathcal{G} / \mathcal{T}$ is locally finitely presented, the quotient functor $q: \mathcal{G} \longrightarrow \mathcal{G} / \mathcal{T}$ preserves finitely presented objects and $q(\mathcal{S})$ is a set of (finitely presented) generators of $\mathcal{G} / \mathcal{T}$.

Proof. Let $X$ be a finitely presented object of $\mathcal{G}$ and let $\left(Y_{i}\right)_{i \in I}$ a direct system in $\mathcal{G} / \mathcal{T}$. Using the adjunction $q \vdash \iota$ and the fact that $\mathcal{T}$ is of finite type, we get a sequence of isomorphisms

$$
\begin{aligned}
& \underset{\lim }{\longrightarrow} \operatorname{Hom}_{\mathcal{G} / \mathcal{T}}\left(q(X), Y_{i}\right) \cong \lim _{\longrightarrow} \operatorname{Hom}_{\mathcal{G}}\left(X, \iota\left(Y_{i}\right)\right) \cong \operatorname{Hom}_{\mathcal{G}}\left(X, \underline{\lim } \iota\left(Y_{i}\right)\right) \cong \operatorname{Hom}_{\mathcal{G}}\left(X, \iota\left(\underline{\lim }\left(Y_{i}\right)\right)\right) \cong \\
& \operatorname{Hom}_{\mathcal{G} / \mathcal{T}}\left(q(X), \lim _{i} Y_{i}\right)
\end{aligned}
$$


whose composition coincides with the canonical morphism

$$
\underset{\lim }{\longrightarrow} \operatorname{Hom}_{\mathcal{G} / \mathcal{T}}\left(q(X), Y_{i}\right) \longrightarrow \operatorname{Hom}_{\mathcal{G} / \mathcal{T}}\left(q(X), \lim _{i} Y_{i}\right)
$$

It follows that $q(X)$ is a finitely presented object of $\mathcal{G} / \mathcal{T}$. Therefore $q$ preserves finitely presented objects.

On the other hand, each object of $\mathcal{G} / \mathcal{T}$ is of the form $q(M)$, with $M \in \mathcal{G}$. If we put $M=\lim X_{i}$, with the $X_{i}$ finitely presented, then we get $q(M)=\lim _{q} q\left(X_{i}\right)$ since the functor $q$ preserves direct limits. It follows that each object of $\mathcal{G} / \mathcal{T}$ is a direct limit of finitely presented objects.

Finally, for $M$ as above, we have an epimorphism $\coprod_{j \in J} S_{j} \rightarrow M$ in $\mathcal{G}$, where the $S_{j}$ are in $\mathcal{S}$. Then we get an epimorphism $\coprod_{j \in J} q\left(S_{j}\right) \rightarrow q(M)$ in $\mathcal{G} / \mathcal{T}$. This shows that $q(\mathcal{S})$ is a set of finitely presented generators of $\mathcal{G} / \mathcal{T}$ and, hence, that $\mathcal{G} / \mathcal{T}$ is locally finitely presented.

Recall that if $\mathcal{X}$ is any set of objects in $\mathcal{G}$ and $M$ is another object, then the trace of $\mathcal{X}$ on $M$ is the sum of all the images of morphisms $f: X \longrightarrow M$, with $X \in \mathcal{X}$. Given a hereditary torsion class $\mathcal{T}$ in $\mathcal{G}$, with torsion radical $t$, and a class $\mathcal{X}$ of objects in $\mathcal{G}$, we shall denote by $\overline{\mathcal{X}}$ the class of objects $X / t(X)$, with $X \in \mathcal{X}$. Also, given an object $X \in \mathcal{G}$, we denote by $\mathcal{T}_{X}$ the class of objects $T \in \mathcal{T}$ which are epimorphic image of $X$.

Proposition 2.5. Let $I$ be a downward directed preordered set without minimum and let $\mathcal{G}$ be a locally finitely presented Grothendieck category admitting a set $\mathcal{S}$ of finitely presented generators decomposed as a union $\mathcal{S}=\bigcup_{i \in I} \mathcal{S}_{i}$ of nonempty subsets. Let us denote by $\operatorname{tr}_{j}(M)$ the trace of $\bigcup_{k \leq j} \mathcal{S}_{k}$ in $M$, for each $M \in \mathcal{G}$ and each $j \in I$. Suppose that $\mathcal{T}$ is a hereditary torsion class of finite type, with torsion radical $t$, satisfying the following conditions:

1. For each $i \in I$ and each $X \in \mathcal{S}_{i}$, the set of indices $j<i$ such that either $\operatorname{Hom}_{A}(X, ?)_{\mid \overline{\mathcal{S}}_{j}} \neq 0$ or $\operatorname{Ext}_{\mathcal{G}}^{1}(?, ?)_{\mid \mathcal{T}_{X} \times \overline{\mathcal{S}}_{j}} \neq 0$ is finite.

2. For each pair of indices $j<i$ in $I$ and each $X \in \mathcal{S}_{i}$, one has that $X / \operatorname{tr}_{j}(X) \in \mathcal{T}$.

Then $\mathcal{G} / \mathcal{T}$ is locally finitely presented and contains no nonzero flat object.

Proof. By proposition 2.4 $\mathcal{G} / \mathcal{T}$ is locally finitely presented and $q(\mathcal{S})$ is a set of finitely presented generators of $\mathcal{G} / \mathcal{T}$.

We shall prove that $q(\mathcal{S})=\bigcup_{i \in I} q\left(\mathcal{S}_{i}\right)$ is a densely $I$-stratified set of generators of $\mathcal{G} / \mathcal{T}$ and the result will follow from proposition 2.2. By condition 1, given an index $i \in I$ and an object $X \in \mathcal{S}_{i}$, the set $I_{X}$ of indices $j<i$ such that $\operatorname{Hom}_{\mathcal{G}}(X, ?)_{\mid \overline{\mathcal{S}}_{j}}=0=\operatorname{Ext}_{\mathcal{G}}^{1}(?, ?)_{\mid \mathcal{T}_{X} \times \overline{\mathcal{S}}_{j}}$ is cofinite within the set of indices $j \in I$ such that $j<i$. We claim that if $j \in I_{X}$, then $\operatorname{Hom}_{\mathcal{G} / \mathcal{T}}(q(X), q(Y))=0$, for all $Y \in \mathcal{S}_{j}$. To see that, take $Y \in \mathcal{S}_{j}$ and apply the functor $\operatorname{Hom}_{\mathcal{G}}(X, ?)$ to the exact sequence

$$
0 \rightarrow Y / t(Y) \stackrel{\mu}{\longrightarrow}(\iota \circ q)(Y) \stackrel{p}{\longrightarrow} T \rightarrow 0
$$

where $\mu$ comes from the unit map of the adjunction $q \vdash \iota$. We know that $T \in \mathcal{T}$. If now $f: X \longrightarrow T$ is any morphism, then $\operatorname{Im}(f) \in \mathcal{T}_{X}$ and hence $\operatorname{Ext}_{\mathcal{G}}^{1}(\operatorname{Im}(f), Y / t(Y))=0$. But then the inclusion $\operatorname{Im}(f) \hookrightarrow T$ factors through $p$. This implies that $\operatorname{Im}(f)=0$ since $\operatorname{Hom}_{\mathcal{G}}(\operatorname{Im}(f),(\iota \circ q)(Y))=0$. Therefore we have $\operatorname{Hom}_{\mathcal{G}}(X, T)=0$ and, hence, we also have $0=\operatorname{Hom}_{\mathcal{G}}(X,(\iota \circ q)(Y)) \cong \operatorname{Hom}_{\mathcal{G} / \mathcal{T}}(q(X), q(Y))$ because $\operatorname{Hom}_{\mathcal{G}}(X, Y / t(Y))=0$ by hypothesis.

The last paragraph shows that, given $i \in I$ and $X \in \mathcal{S}_{i}$, the set of indices $j<i$ such that $\operatorname{Hom}_{\mathcal{G} / \mathcal{T}}(q(X), ?)_{\mid q\left(\mathcal{S}_{j}\right)} \neq 0$ is finite. Therefore $q(\mathcal{S})$ satisfies condition 2 of definition 1

We now prove that $q(\mathcal{S})$ satisfies condition 3 of in that definition. Indeed if $j<i$ then, for each $X \in \mathcal{S}_{i}$, we consider the canonical morphism in $\mathcal{G}$

$$
f: \coprod_{S \in \bigcup_{k \leq j} \mathcal{S}_{k}} S^{\left(\operatorname{Hom}_{\mathcal{G}}(S, X)\right)} \longrightarrow X,
$$

whose image is precisely $\operatorname{tr}_{j}(X)$. By condition 2, we know that $\operatorname{Coker}(f) \in \mathcal{T}$. But then the induced morphism in $\mathcal{G} / \mathcal{T}$ 


$$
\coprod_{S \in \bigcup_{k \leq j} \mathcal{S}_{k}} q(S)^{\left(\operatorname{Hom}_{\mathcal{G}}(S, X)\right)} \cong q\left(\coprod_{S \in \bigcup_{k \leq j} \mathcal{S}_{k}} S^{\left(\operatorname{Hom}_{\mathcal{G}}(S, X)\right)}\right) \stackrel{q(f)}{\longrightarrow} q(X),
$$

is an epimorphism, because $q$ is exact and vanishes on $\mathcal{T}$.

\section{Quotient categories of graded modules without flat objects}

All throughout this section $A=\oplus_{n>0} A_{n}$ is a positively graded ring. Further hypotheses will be imposed on it when stating the main results. We denote by $A-G r$ the category of ( $\mathbb{Z}$-)graded $A$-modules. It is a Grothendieck category. For each $n \in \mathbb{Z}$, there is a canonical automophism ?[n]:A-Gr $\cong A-G r$, called the $n$-th shift. For each $M \in A-G r, M[n]$ is the graded $A$-module with the same underlying (ungraded) $A$-module as $M$, but with grading given by $M[n]_{i}=M_{n+i}$. It is well-known (cf. 23]) that $A-G r$ is locally finitely presented, with $\{A[n]: n \in \mathbb{Z}\}$ as a set of finitely generated projective generators.

We will use some standard facts about Hom and Ext for graded modules (see, e.g., 23, Chapter I]). If $M$ and $N$ are graded $A$-modules and we put $\operatorname{HOM}_{A}(M, N)=\oplus_{n \in \mathbb{Z}} \operatorname{Hom}_{A-G r}(M, N[n])$, then $\operatorname{HOM}_{A}(M, N)$ is a graded abelian group with $\operatorname{HOM}_{A}(M, N)_{n}=\operatorname{Hom}_{A-G r}(M, N[n])$, for each $n \in \mathbb{Z}$. Then one gets a covariant functor $\operatorname{HOM}_{A}(M, ?): A-G r \longrightarrow \mathbb{Z}-G r$. Its right derived functors are denoted by $\operatorname{EXT}_{R}^{i}(M, ?)(i \geq 0)$. It is immediate to see that $\operatorname{EXT}_{A}^{i}(M, N)=\oplus_{n \in \mathbb{Z}} \operatorname{Ext}_{A-G r}^{i}(M, N[n]) \cong$ $\oplus_{n \in \mathbb{Z}} \operatorname{Ext}_{A-G r}^{i}(M[-n], N)$, which is a graded abelian group with $\operatorname{EXT}_{A}^{i}(M, N)_{n}=\operatorname{Ext}_{A-G r}^{i}(M, N[n]) \cong$ $\operatorname{Ext}_{A-G r}^{i}(M[-n], N)$, for each $n \in \mathbb{Z}$.

On the other hand, when one forgets the grading, $\operatorname{HOM}_{A}(M, N)$ is a subgroup of $\operatorname{Hom}_{A}(M, N)$ which coincides with it in case $M$ is finitely generated (see [23, Corollary I.2.11]). If, in addition, $M$ has a projective resolution

$$
\ldots \rightarrow P^{-n} \rightarrow \ldots \rightarrow P^{-1} \rightarrow P^{0} \rightarrow M \rightarrow 0
$$

such that $P^{-i}$ is finitely generated for $i=0,1, \ldots, r$, then the two compositions of functors

$$
A-G r \stackrel{\text { forgetful }}{\longrightarrow} A-\operatorname{Mod} \stackrel{\operatorname{Ext}_{A}^{i}(M, ?)}{\longrightarrow} \mathbb{Z}-\operatorname{Mod} A-G r \stackrel{\operatorname{EXT}_{A}^{i}(M, ?)}{\longrightarrow} \mathbb{Z}-G r \stackrel{\text { forgetful }}{\longrightarrow} \mathbb{Z}-\operatorname{Mod}
$$

are naturally isomorphic, for all $0 \leq i<r$.

If $A=\oplus_{n \geq 0} A_{n}$ is any positively graded ring, then we have a homomorphism of graded rings $\pi: A \longrightarrow$ $A_{0}$ mapping each $a \in A$ onto its 0 -homogeneous component $a_{0}$ (here we are viewing $A_{0}$ as a graded ring concentrated in degree 0 ). Then each $A_{0}$-module, and in particular $A_{0}$ itself, becomes canonically a graded $A$-module concentrated in degree 0 .

Recall that a $T \in A-G r$ is called finitely graded when $T_{n}=0$, for all but finitely many integers $n$. We shall say that $T$ is virtually finitely graded when each finitely generated (or cyclic) graded submodule of $T$ is finitely graded. All throughout this section $\mathcal{T}$ will denote the class of virtually finitely graded modules. Recall (cf. [23]) that a hereditary torsion theory in $A-G r$ is called rigid when $T[n] \in \mathcal{T}$, for all $T \in \mathcal{T}$ and $n \in \mathbb{Z}$.

Lemma 3.1. Suppose that there is an integer $k>0$ such that $A_{\geq m}:=\oplus_{n \geq m} A_{n}$ is finitely generated as left ideal, for all $m \geq k$. The class $\mathcal{T}$ is a rigid hereditary torsion class in $A-G r$. Moreover, if $A_{\geq 1}:=\oplus_{n \geq m} A_{1}$ is finitely presented as a left ideal and $\iota: A-G r / \mathcal{T} \longrightarrow A-G r$ is the section functor, then:

1. $\operatorname{Im}(\iota)$ consists of the graded A-modules $Y$ such that $\operatorname{Hom}_{A}\left(A_{0}, Y\right)=0=\operatorname{Ext}_{A}^{1}\left(A_{0}, Y\right)$

2. $\mathcal{T}$ is of finite type.

Proof. The class $\mathcal{T}$ is clearly closed under taking subobjects, quotients and arbitrary coproducts, even without the existence of the integer $k$ of the statement. Note that an alternative description of a $T \in \mathcal{T}$ is that it is a graded module such that each of its homogeneous elements is annihilated by $A_{\geq m}$, for some 
$m \geq 0$. Suppose now that $0 \rightarrow T^{\prime} \longrightarrow M \longrightarrow T \rightarrow 0$ is an exact sequence in $A-G r$, with $T, T^{\prime} \in \mathcal{T}$. For each homogeneous element $x \in M$, the cyclic graded module $A x+T^{\prime} / T^{\prime}$ is isomorphic to a graded submodule of $T$. Then there exists an integer $m>0$ such that $A_{\geq m} x \subset T^{\prime}$. If we choose $m \geq k$, where $k$ is the integer in the statement, then $A_{>m} x$ is a finitely generated graded submodule of $T^{\prime}$. We get that $A_{\geq m} x$ is finitely graded and $A x / A_{\geq m} x$ is obviously finitely graded. It follows that $A x$ is finitely graded, and hence $\mathcal{T}$ is a hereditary torsion class in $A-G r$, which is clearly rigid.

Suppose now that $A_{\geq 1}$ is finitely presented as a left ideal and let us prove assertions 1 and 2. If $Y \in \operatorname{Im}(\iota)$ then $\operatorname{Hom}_{A-G r}\left(A_{0}[n], Y\right)=0=\operatorname{Ext}_{A-G r}^{1}\left(A_{0}[n], Y\right)$ since $A_{0}$ is in $\mathcal{T}$ when we view it as a graded module concentrated in degree zero. But the comments preceding this lemma show that we have equalities $\operatorname{Hom}_{A}\left(A_{0}, Y\right)=\operatorname{HOM}_{A}\left(A_{0}, Y\right)$ and $\operatorname{Ext}_{A}^{1}\left(A_{0}, Y\right)=\operatorname{EXT}_{A}^{1}\left(A_{0}, Y\right)$ and, hence, we get that $\operatorname{Hom}_{A}\left(A_{0}, Y\right)=0=\operatorname{Ext}_{A}^{1}\left(A_{0}, Y\right)$.

Conversely, suppose that $\operatorname{Hom}_{A}\left(A_{0}, Y\right)=0=\operatorname{Ext}_{A}^{1}\left(A_{0}, Y\right)$. Then $\operatorname{Hom}_{A}(T, Y)=0$, for all $T \in \mathcal{T}$ (i.e. $\left.Y \in \mathcal{T}^{\perp}\right)$. Indeed, if $f: T \longrightarrow Y$ is a nonzero morphism in $A-G r$, then $\operatorname{Im}(f) \in \mathcal{T}$. Then each finitely generated submodule of $\operatorname{Im}(f)$ is finitely graded and, hence, it contains a nonzero homogeneous element annihilated by $A_{\geq 1}$. We then obtain a nonzero morphism $A_{0}[n] \cong \frac{A}{A_{\geq 1}}[n] \longrightarrow \operatorname{Im}(f) \hookrightarrow Y$, which contradicts the fact that $\operatorname{Hom}_{A}\left(A_{0}, Y\right)=0$.

By last paragraph, the task reduces to prove that if $Y \in \mathcal{T}^{\perp}$ is such that $\operatorname{Ext}_{A}^{1}\left(A_{0}, Y\right)=0$, then $\operatorname{Ext}_{A-G r}^{1}(T, Y)=0$ holds for all $T \in \mathcal{T}$. Let $T \in \mathcal{T}$ be any object, which we express as a direct union $\bigcup_{i \in I} T_{i}$ of its finitely generated graded submodules. Then we have an exact sequence in $A-G r$

$$
0 \rightarrow T^{\prime} \longrightarrow \bigsqcup_{i \in I} T_{i} \stackrel{\pi}{\longrightarrow} T \rightarrow 0,
$$

where $\pi$ is the canonical projection onto the direct limit. Then we get an exact sequence of abelian groups

$$
0=\operatorname{Hom}_{A-G r}\left(T^{\prime}, Y\right) \longrightarrow \operatorname{Ext}_{A-G r}^{1}(T, Y) \longrightarrow \operatorname{Ext}_{A-G r}^{1}\left(\amalg_{i \in I} T_{i}, Y\right) .
$$

Bearing in mind that $\operatorname{Ext}_{A-G r}^{1}(?, Y): A-G r \longrightarrow A b$ takes coproducts to products, our task is reduced to check that $\operatorname{Ext}_{A-G r}^{1}(T, Y)=0$, whenever $T \in \mathcal{T}$ is finitely generated. By definition of $\mathcal{T}$, if $T \in \mathcal{T}$ is finitely generated, then it is concentrated in degrees $\{r, r+1, \ldots, s\}$, for some integers $r<s$. Then the chain

$$
0 \subset T_{s} \subset\left(T_{s-1} \oplus T_{s}\right) \subset \ldots \subset\left(T_{r+1} \oplus \ldots \oplus T_{s}\right) \subset \oplus_{r \leq k \leq s} T_{k}=T
$$

is a finite ascending chain in $A-G r$ on which each quotient of a member by the previous one is annihilated by $A_{\geq 1}$ and is concentrated in just one degree. The proof then reduces to the case when $T=X[n]$, for some $A_{0}$-module $X$ (viewed as a graded $A$-module concentrated in degree 0 ) and some $n \in \mathbb{Z}$. Fix such $X$ and $n$ and consider an exact sequence of $A_{0}$-modules

$$
0 \rightarrow Z \longrightarrow A_{0}^{(H)} \longrightarrow X \rightarrow 0
$$

which we view as a sequence of graded $A$-modules which are annihilated by $A_{\geq 1}$. We then get an exact sequence of abelian groups

$$
0=\operatorname{Hom}_{A-G r}(Z[n], Y) \longrightarrow \operatorname{Ext}_{A-G r}^{1}(X[n], Y) \longrightarrow \operatorname{Ext}_{A-G r}^{1}\left(A_{0}^{(H)}[n], Y\right) .
$$

The proof of assertion 1 will be finished if we prove that $\operatorname{Ext}_{A-G r}^{1}\left(A_{0}[n], Y\right)=0$, for all $n \in \mathbb{Z}$, since $\operatorname{Ext}_{A-G r}^{1}(?, Y)$ takes coproducts to products. But $\operatorname{Ext}_{A-G r}^{1}\left(A_{0}[n], Y\right)$ is just the $-n$-th homogeneous component of $\operatorname{EXT}_{A}^{1}\left(A_{0}, Y\right)=\operatorname{Ext}_{A}^{1}\left(A_{0}, Y\right)$, which is zero by hypothesis.

In order to prove assertion 2 , note that $\mathcal{T}$ is of finite type if, and only if, the class $\operatorname{Im}(\iota)$ is closed under taking direct limits in $A-G r$. Bearing in mind that $A_{0}$ admits a projective resolution

$$
\ldots \rightarrow P^{-2} \rightarrow P^{-1} \rightarrow P^{0} \rightarrow A_{0} \rightarrow 0
$$

in $A-G r$, where $P^{-i}$ is finitely generated for $i=0,1,2$, we get that the composition 


$$
A-G r \stackrel{\text { forgetful }}{\longrightarrow} A-\operatorname{Mod} \stackrel{\operatorname{Ext}_{A}^{1}\left(A_{0}, ?\right)}{\longrightarrow} \mathbb{Z}-\operatorname{Mod}
$$

preserves direct limits. This together with assertion 1 show that $\operatorname{Im}(\iota)$ is closed under direct limits.

We are now ready to prove the main result of this section.

Theorem 3.2. Let $A=\oplus_{n \geq 0} A_{n}$ be a positively graded ring with the property that $A_{\geq 1}$ is finitely presented and $A_{\geq n}$ is finitely generated, as left ideals, for all $n>>0$. Let $\mathcal{T}$ be the class of virtually finitely graded left $A$-modules and the associated torsion radical. The category $A-G r / \mathcal{T}$ is locally finitely presented and, in case $\operatorname{Ext}_{A}^{1}\left(A_{0}, A / t(A)\right)=0$, it has no nonzero flat objects.

Proof. By lemma 3.1 we know that $\mathcal{T}$ is a hereditary torsion class of finite type, and then, by proposition 2.4. we know that $A-G r / \mathcal{T}$ is locally finitely presented.

Let us put $I=\mathbb{Z}$ and $\mathcal{S}_{n}=\{A[n]\}$, for each $n \in \mathbb{Z}$. We shall prove that the two conditions of proposition 2.5 hold for $\mathcal{S}=\bigcup_{n \in \mathbb{Z}} \mathcal{S}_{n}$. Note that $\overline{\mathcal{S}}_{n}=\left\{\frac{A}{t(A)}[n]\right\}$, for each $n \in \mathbb{Z}$. We have that $\operatorname{Hom}_{A-G r}\left(A[m], \frac{A}{t(A)}[n]\right) \cong\left(\frac{A}{t(A)}\right)_{n-m}$, which is zero when $n<m$. Then the set of indices mentioned in condition 1 of proposition 2.5 is empty in this case.

On the other hand, if $m<n$ are integers then we have $\operatorname{tr}_{m}(A[n])=t r_{m-n}(A)[n]$ and hence $\frac{A[n]}{t r_{m}(A[n])} \cong$ $\frac{A}{t r_{m-n}(A)}[n]$ and, in order to check condition 2 of proposition 2.5. it is enough to check that $A / \operatorname{tr}_{-r}(A) \in \mathcal{T}$, for all $r>0$. But the trace of $\bigcup_{k \leq-r} \mathcal{S}_{k}=\{A[k]: k \leq-r\}$ in $A$ is precisely $A_{\geq r}=\oplus_{n \geq r} A_{n}$, and hence $A / \operatorname{tr}_{-r}(A)=A / A_{\geq r}$. This is a finitely graded $A$-module and, hence, it is in $\mathcal{T}$.

\section{The category of quasicoherent sheaves on a projective scheme has no nonzero flat objects}

Recall that if $A=\oplus_{n \geq 0} A_{n}$ is a positively graded commutative ring, then $\operatorname{Proj}(A)$ is the set of graded prime ideals $\mathbf{p}$ of $A$ such that $A_{\geq 1} \not \subset \mathbf{p}$. It is a scheme (cf. [14, Chapter II]) whose underlying topology is the Zariski one. The (Grothendieck) category of quasicoherent sheaves on $\operatorname{Proj}(A)$ is our next objective. When $M$ is a graded $A$-module and $\mathbf{p} \in \operatorname{Proj}(A)$, we shall denote by $M_{(\mathbf{p})}$ the graded localization of $M$ at $\mathbf{p}$. It consists of the fractions $\frac{x}{s}$, where $x \in M$ and $s \in A \backslash \mathbf{p}$ are homogeneous elements with the same degree.

Lemma 4.1. Let $A=\oplus_{n \geq 0} A_{n}$ be a commutative positively graded ring which is finitely generated by $A_{1}$ as an $A_{0}$-algebra. The class $\mathcal{T}$ of virtually finitely graded $A$-modules is a rigid hereditary torsion class in $A-G r$ and it consits of those $M \in A-G r$ such that $M_{(\mathbf{p})}=0$, for all $\mathbf{p} \in \operatorname{Proj}(A)$.

Proof. If $\left\{x_{0}, \ldots, x_{n}\right\}$ is a set of elements of $A_{1}$ which generates $A$ as an $A_{0}$-algebra, then the graded ideal $A_{\geq m}$ is generated by all monomials of degree $m$ in the $x_{i}$. It follows that $A_{\geq m}$ is a finitely generated ideal, for each $m>0$. By lemma 3.1, we get that $\mathcal{T}$ is a rigid hereditary torsion class in $A-G r$.

To prove the final statement, due to the exactness of localization, we just need to check that a cyclic graded $A$-module $M$ is finitely graded if, and only if, $M_{(\mathbf{p})}=0$ for all $\mathbf{p} \in \operatorname{Proj}(A)$. For the 'only if' part note that if $M$ is finitely graded, then there is a power $\left(A_{\geq 1}\right)^{m}$ which annihilates $M$. Choosing an element $s_{\mathbf{p}} \in A_{\geq 1} \backslash \mathbf{p}$, we get that $s_{\mathbf{p}}^{m} M=0$ and therefore $M_{(\mathbf{p})}=0$, for each $\mathbf{p} \in \operatorname{Proj}(A)$.

For the 'if' part, suppose $M=A x$, where $x$ is a homogenous element of degree $r$. Then, applying the shift ?[-r]:A-Gr $\longrightarrow A-G r$, we can and shall assume, without loss of generality, that $\operatorname{deg}(x)=0$. Then $M$ is isomorphic to $A / I$, for some graded ideal $I$ of $A$. Saying that $M_{(\mathbf{p})}=0$, for all $\mathbf{p} \in \operatorname{Proj}(A)$ is equivalent to saying that the graded ring $A / I$ has $\operatorname{Proj}(A / I)=\emptyset$. This means that $(A / I)_{\geq 1}=\frac{A_{\geq 1}+I}{I}$ is contained in all the graded prime ideals of $A / I$. That is, the homogeneous elements of $(A / I)_{\geq 1}$ are all nilpotent, and then $(A / I)_{\geq 1}$ is a nilpotent ideal of $A / I$ since $(A / I)_{\geq 1}$ is a finitely generated ideal of $A / I$. This means that there is a large enough integer $m>0$ such that $\left(A_{\geq 1}\right)^{n} \subseteq I$, for all $n \geq m$. But, 
due to the fact that $A_{1}$ generates $A$ as an $A_{0}$-algebra, we have an equality $A_{n}=A_{1}^{n}$, for each $n>0$. It follows that $A_{n} \subset I$, and hence $(A / I)_{n}=0$, for all $n \geq m$. Then $M \cong A / I$ is finitely graded.

For any (algebraic) scheme $X$, we denote by $Q \operatorname{coh}(X)$ the category of quasi-coherent sheaves on $X$. The following result, essentially due to Serre, is standard. For the convenience of the reader, we give a summary of the proof essentially derived from Murfet's notes (see [21] and [22]).

Theorem 4.2 (Serre). Let $A=\oplus_{n \geq 0} A_{n}$ be a positively graded commutative algebra which is finitely generated by $A_{1}$ as an $A_{0}$-algebra, let $X=\operatorname{Proj}(A)$ be the associated projective scheme and let $\mathcal{T}$ be the class of virtually finitely graded $A$-modules. There is an equivalence of categories $A-G r / \mathcal{T} \cong Q \operatorname{coh}(X)$.

Proof. Using the notation of [14, Chapter II, Section 5], the assignment $M \rightsquigarrow \tilde{M}$ gives a functor $(-)^{\sim}$ : $A-G r \longrightarrow O_{X}-$ Mod which is exact (see, e.g., [22, Lemma 2]) and, by definition of quasicoherent sheaf, this functor has $Q \operatorname{coh}(X)$ as its essential image. Then the induced functor $p: A-G r \longrightarrow Q \operatorname{coh}(X)$ is also exact because $Q \operatorname{coh}(X)$ is a full subcategory of $O_{X}-\operatorname{Mod}$.

On the other hand, there is an additive functor $\Gamma_{*}: O_{X}-\operatorname{Mod} \longrightarrow A-G r$ which is right adjoint to $(-)^{\sim}$ (see [21, Proposition 2]). We shall denote by $\epsilon:(-)^{\sim} \circ \Gamma_{*} \longrightarrow 1_{O_{X}-M O d}$ and $\eta: 1_{A-G r} \longrightarrow \Gamma_{*} \circ(-) \sim$ the counit and the unit of this adjunction, respectively. It follows that $p$ is left adjoint to the restriction $j:=\Gamma_{* \mid Q \operatorname{coh}(X)}: Q \operatorname{coh}(X) \longrightarrow A-G r$. By [14, Proposition II.5.15], we know that the counit of this adjunction is the identity.

Now proposition 2.3 tells us that $Q \operatorname{coh}(X)$ is equivalent to $A-G r / \operatorname{Ker}(p)$ and our task reduces to check that $\operatorname{Ker}(p)=\mathcal{T}$. But, by the properties of sheaves, we know that $\tilde{M}=0$ if, and only if, each stalk $\tilde{M}_{\mathbf{p}}$ is zero. By [14, Proposition II.5.11], this is equivalent to saying that $M_{(\mathbf{p})}=0$, for all $\mathbf{p} \in \operatorname{Proj}(A)$. The preceding lemma states that this is equivalent to saying that $M \in \mathcal{T}$.

Remark 4.3. In the theory of (algebraic schemes) there is a classical concept of flat sheaf, which we call here geometric flat. Namely, if $X$ is a scheme with structural sheaf of rings $O_{X}$, then a sheaf $\mathcal{F}$ of $O_{X}$-modules is geometrically flat when the stalk $\mathcal{F}_{x}$ is a flat $O_{X, x}$-module, for each point $x \in X$. For this reason, a flat object in a Grothendieck category $\mathcal{G}$ will be called categorical flat in the particular case when $\mathcal{G}=Q \operatorname{coh}(X)$, for a scheme $X$.

The main result of this section is the following.

Theorem 4.4. Let $A=\oplus_{n \geq 0} A_{n}$ be a positively graded commutative ring which is finitely generated by $A_{1}$ as an $A_{0}$-algebra, let $I$ be the (graded) ideal of $A$ consisting of those a $\in A$ such that $A_{1}^{n} a=0$, for some $n=n(a) \in \mathbb{N}$, and let $X=\operatorname{Proj}(A)$ be the associated projective scheme. If the two following conditions hold, then $Q \operatorname{coh}(X)$ has no nonzero categorical flat object:

1. The ideal $A_{\geq 1}$ is finitely presented.

2. $\operatorname{Ext}_{A}^{1}\left(A_{0}, A / I\right)=0$

Proof. Note that a graded $A$-module is finitely graded if, and only if, it is annihilated by $A_{n}=A_{1}^{n}$, for some $n \geq 0$. This shows that $I=t(A)$, where $t$ is the torsion radical associated to $\mathcal{T}$.

By the proof of lemma 4.1, we know that $A_{\geq m}$ is finitely generated, for all $m \geq 0$. Then, by theorem 3.2. we know that $A-G r / \mathcal{T}$ does not have nonzero flat objects. Finally, by Serre's theorem 4.2, we get the result.

We refer to [20] or [17] for the terminology used in the following result.

Corollary 4.5. Let $A=\oplus_{n \geq 0} A_{n}$ be a positively graded commutative Noetherian domain which is finitely generated by $A_{1}$ as an $A_{0}$-algebra, suppose that $A_{\geq 1}$ has height $\geq 2$ and let $X=\operatorname{Proj}(A)$ be the associated projective scheme. If $A$ satisfies one of the following two conditions (as an ungraded ring), then $Q \operatorname{coh}(X)$ has no nonzero categorical flat objects. 


\section{A is integrally closed}

\section{A is Cohen-Macaulay}

Proof. In both cases the ideal $I$ of theorem 4.4 is zero.

1) If $A$ is integrally closed, then $A$ satisfies Serre condition $S_{2}$ (see [31, Chapter VII, Section 6]) for the definition and the result). Now proposition VII.6.8 in 31 says that $A / A_{\geq 1}=A_{0}$ satisfies the property that $\operatorname{Hom}_{A}\left(A_{0}, E^{0}(A) \oplus E^{1}(A)\right)=0$, where $0 \rightarrow A \longrightarrow E^{0}(A) \longrightarrow E^{1}(A)$ is the initial part of the minimal injective resolution of $A$ in $A$ - Mod. It follows immediately that $\operatorname{Ext}_{A}^{1}\left(A_{0}, A\right)=0$ and, hence, theorem 4.4 applies.

2) If $A$ is Cohen-Macaulay, then $\operatorname{depth}\left(A_{\geq 1}, A\right)$ coincides with the height $\operatorname{ht}\left(A_{\geq 1}\right)$ (see [17, Theorem VI.3.14]). By definition of the depth, this means that $0=\operatorname{Ext}_{A}^{i}\left(A / A_{\geq 1}, A\right)=\operatorname{Ext}_{A}^{i}\left(A_{0}, A\right)$, for all $i<\operatorname{ht}\left(A_{\geq 1}\right)$. In particular $\operatorname{Ext}_{A}^{1}\left(A_{0}, A\right)=0$ and theorem 4.4 applies again.

Recall that if $R$ is a commutative ring and $n>0$ is an integer, then the projective $n$-space over $R$ is $\mathbf{P}^{n}(R)=\operatorname{Proj}\left(R\left[x_{0}, \ldots, x_{n}\right]\right)$, where the $x_{i}$ are variables over $R$ of degree 1 .

Corollary 4.6. $Q \operatorname{coh}\left(\mathbf{P}^{n}(R)\right)$ does not have nonzero categorical flat objects, for any commutative ring $R$ and any integer $n>0$.

Proof. Put $A:=R\left[x_{0}, x_{1}, \ldots, x_{n}\right]$ with its canonical grading. Here $A_{\geq 1}=\sum_{0<i<n} A x_{i}$ is the ideal generated by $\left\{x_{0}, x_{1}, \ldots, x_{n}\right\}$ and the ideal $I$ of theorem 4.4 is zero. Moreover, we have $A_{0}=R$, viewed as $A$-module in the usual way and $A_{1}=\sum_{0 \leq i \leq n} R x_{i}$. Therefore $A$ is generated by $A_{1}$ as an $A_{0}$-algebra. The result will follow from theorem 4.4 once we check that $\operatorname{Ext}_{A}^{1}(R, A)=0$. But this is well-known. It is a straightforward consequence of [4, Chapter VIII, exercise 7] or, by finite induction, from [26, Theorem $9.37]$.

\section{Module categories modulo locally finite dimensional ones}

In this section we fix a commutative ring $R$ with 1 and the term 'algebra' will mean (not necessarily unital, but always associative) ' $R$-algebra'. An algebra with enough idempotents is an associative algebra $A$ on which a distinguished family of nonzero orthogonal idempotents $\left(e_{i}\right)_{i \in I}$ has been fixed, satisfying that $\oplus_{i \in I} e_{i} A=A=\oplus_{i \in I} A e_{i}$ as an $R$-module. We assume all the time that $R e_{i}=e_{i} R$ is free of rank 1 over $R$ generated by $e_{i}$ and put $B=\oplus_{i \in I} R e_{i}$, which is a commutative subalgebra of $A$. We will assume that $A$ is augmented (with respect to $B$ ). This means that the inclusion $B \hookrightarrow A$ is a section in the category of algebras or, equivalently, that there exists a two-sided ideal $J$ of $A$ such that $A=B \oplus J$ as $B$-bimodules. Although $J$ is not unique, we will fix one and the corresponding decomposition $A=B \oplus J$ will be called the Wedderburn-Malcev type decomposition of $A$. Over such an algebra $A$, a (left) module $M$ will be always considered to be unitary, i.e. $A M=M$ or, equivalently, it admits a decomposition $M=\oplus_{i \in I} e_{i} M$ as an $R$-module. The corresponding category is denoted by $A-\operatorname{Mod}$ and is a Grothendieck category with $\left\{A e_{i}: i \in I\right\}$ as a set of finitely generated projective generators. We call an $A$-modulo $M$ torsion (with respect to the given Wedderburn-Malcev decomposition) when each element of $M$ is annihilated by some power $J^{n}$. In this section, unless explicitly said otherwise, we denote by $\mathcal{T}$ the full subcategory of $A-$ Mod consisting of torsion $A$-modules.

The following is the analogous of lemma 3.1 in this context.

Lemma 5.1. Let $A$ an algebra with enough idempotents as above, with $\left(e_{i}\right)_{i \in I}$ as a distinguished family of nonzero orthogonal idempotents, let $A=B \oplus J$ be a fixed Wedderburn-Malcev type decomposition, where $B=\oplus_{i \in I} R e_{i}$, and let $\mathcal{T}$ be the corresponding class of torsion A-modules. Suppose that, for each $i \in I$, there is an integer $k(i)>0$ such that $J^{m} e_{i}$ is a finitely generated left ideal, for all $m \geq k(i)$. Then $\mathcal{T}$ is a hereditary torsion class in $A-M o d$. Moreover, if Je $e_{i}$ is finitely presented as a left ideal, for each $i \in I$ and $\iota: A-\operatorname{Mod} / \mathcal{T} \longrightarrow A-\operatorname{Mod}$ is the section functor, then: 
1. $\operatorname{Im}(\iota)$ consists of the $A$-modules $Y$ such that $\operatorname{Hom}_{A}\left(B e_{i}, Y\right)=0=\operatorname{Ext}_{A}^{1}\left(B e_{i}, Y\right)$, for all $i \in I$

2. $\mathcal{T}$ is of finite type.

Proof. It is entirely similar to that of lemma 3.1. So we just mention the little changes to be made. To see that $\mathcal{T}$ is a hereditary torsion class, only the closure under extensions need a proof. Choosing an exact sequence

$$
0 \rightarrow T^{\prime} \longrightarrow M \longrightarrow T \rightarrow 0
$$

and an element $x \in M$, as in that lemma, we get that $J^{m} x \subseteq T^{\prime}$ for some $m>0$. But there are $i_{1}, \ldots, i_{r} \in I$ such that $x=\sum_{1 \leq t \leq r} e_{i_{t}} x$. Choosing $m \geq \max \left\{k\left(i_{t}\right): t=1, \ldots, r\right\}$, we have that $J^{m} e_{i_{t}} x$ is finitely generated and, hence, also $J^{m} x$ is finitely generated. But then there is a power $J^{p}$ which annihilates $J^{m} x$, so that $J^{p+m} x=0$. Then $M$ is a torsion $A$-module.

As in lemma 3.1 one readily sees that an $A$-module $Y$ is in $\mathcal{T}^{\perp}$ if, and only if, $\operatorname{Hom}_{A}\left(B e_{i}, M\right)=0$ for all $i \in I$. This is because each finitely generated module in $\mathcal{T}$ admits a finite filtration

$$
0 \subsetneq J^{n} x \subsetneq J^{n-1} x \subsetneq \ldots \subsetneq J x \subsetneq A x
$$

where $J^{t} x / J^{t+1} x$ is a $B$-module, for all $t=0,1, \ldots, n$.

Then, again, the proof of assertion 1 reduces to check that if $Y \in \mathcal{T}^{\perp}$ and $\operatorname{Ext}_{A}^{1}\left(B e_{i}, Y\right)=0$, for all $i \in I$, then $\operatorname{Ext}_{A}^{1}(T, Y)=0$, for all $T \in \mathcal{T}$. The corresponding proof of lemma 3.1 can be 'copied', just taking into account that each $B$-module $X$ fills into an exact sequence

$$
0 \rightarrow Z \longrightarrow \oplus_{i \in I} B e_{i}^{\left(H_{i}\right)} \longrightarrow X \rightarrow 0
$$

for some sets $H_{i}$.

Finally, in order to prove assertion 2 , it is enough to observe that, due to the fact that each $J e_{i}$ is assumed to be a finitely presented left ideal, we have a projective resolution of $B e_{i}$ as an $A$-module

$$
\ldots \rightarrow P^{-2} \rightarrow P^{-1} \rightarrow P^{0} \rightarrow B e_{i} \rightarrow 0
$$

where $P^{-i}$ is a finitely generated $A$-module, for $i=0,1,2$. As a consequence, the functors $\operatorname{Hom}_{A}\left(B e_{i}, ?\right)$ and $\operatorname{Ext}_{A}^{1}\left(B e_{i}, ?\right)$ preserve direct limits and, hence, the class $\operatorname{Im}(\iota)$ is closed under taking direct limits in $A-$ Mod.

We now get a wide class of locally finitely presented categories without nonzero flat objects.

Proposition 5.2. Let $A$ be an augmented algebra with enough idempotents, with $\left(e_{i}\right)_{i \in I}$ as a distinguished family of nonzero orthogonal idempotents, let $A=B \oplus J$ be a fixed Wedderburn-Malcev type decomposition, where $B=\oplus_{i \in I} R e_{i}$, and let $\mathcal{T}$ be the corresponding class of torsion A-modules. Assume that, for each $i \in I, J e_{i}$ is finitely presented and $J^{m} e_{i}$ is finitely generated, as left ideals, for all $m>>$. If the following conditions hold, then $A-\operatorname{Mod} / \mathcal{T}$ is a locally finitely presented category without nonzero flat objects:

1. If $\preceq$ the smallest preorder relation in $I$ containing all the pairs $(i, j)$ such that $e_{i} A e_{j} \neq 0$, then $(I, \preceq)$ is downward directed and does not have a minimum.

2. For each $i \in I$, the set of indices $j \prec i$ such that either $\left.\operatorname{Hom}_{A}\left(A e_{i}, \frac{A e_{j}}{t(A) e_{j}}\right)\right) \neq 0$ or

$$
\operatorname{Ext}_{A}^{1}\left(\frac{A e_{i}}{J^{m} e_{i}}, \frac{A e_{j}}{t(A) e_{j}}\right) \neq 0
$$

for some integer $m \geq 0$, is a finite set.

3. For all indices $j \preceq i$, the left $A$-module $\frac{A e_{i}}{\sum_{k \preceq j} A e_{k} A e_{i}}$ is annihilated by some $J^{n}$, with $n>0$. 
Proof. By lemma 5.1 and proposition 2.4, we know that $A-\operatorname{Mod} / \mathcal{T}$ is locally finitely presented.

We put $\mathcal{S}_{i}=\left\{A e_{i}\right\}$, for each $i \in I$, and will prove that conditions 1 and 2 of proposition 2.5 hold. Indeed, if $j<i$ are any indices in $I$, then the trace of $\bigcup_{k \leq j} \mathcal{S}_{k}=\left\{A e_{k}: k \leq j\right\}$ in $A e_{i}$ is precisely $\sum_{k<j} A e_{k} A e_{i}$. Then condition 2 of proposition 2.5 follows from condition 3 in the statement.

Let us take $i \in I$ and take $X=A e_{i}$ to check condition 1 of proposition 2.5. On one side, condition 2 in the statement gives that the set of indices $j \prec i$ such that $\operatorname{Hom}_{A}\left(A e_{i}, \frac{A e_{j}}{t(A) e_{j}}\right) \neq 0$ is finite. On the other hand suppose that $j \prec i$ and $\operatorname{Ext}_{A}^{1}\left(T, \frac{A e_{j}}{t(A) e_{j}}\right) \neq 0$, for some $T \in \mathcal{T}$ which is epimorphic image of $A e_{i}$. Since $T$ is cyclic we have that $J^{m} T=0$, for some $m>0$, so that we have an exact sequence

$$
0 \rightarrow T^{\prime} \longrightarrow \frac{A e_{i}}{J^{m} e_{i}} \longrightarrow T \rightarrow 0
$$

By applying the long exact sequence of $\operatorname{Ext}\left(?, \frac{A e_{j}}{t(A) e_{j}}\right)$ and taking into account that $\operatorname{Hom}_{A}\left(T^{\prime}, \frac{A e_{j}}{t(A) e_{j}}\right)=0$, we get that $\operatorname{Ext}_{A}^{1}\left(\frac{A e_{i}}{J^{m} e_{i}}, \frac{A e_{j}}{t(A) e_{j}}\right) \neq 0$. By condition 2, we know that there are only finitely many such indices $j$. It follows that also condition 1 in proposition 2.5 holds. Therefore $A-\operatorname{Mod} / \mathcal{T}$ does not have nonzero flat objects.

The conditions in last proposition look quite technical. We will see now that the representation theory of quivers with relations provides several examples where the conditions are satisfied. Recall that a quiver (equivalently oriented graph) is a quadruple $Q=\left(Q_{0}, Q_{1}, o, t\right)$, where $Q_{0}$ and $Q_{1}$ are sets, whose elements are calles vertices and arrows respectively, and $o, t: Q_{1} \longrightarrow Q_{0}$ are maps. Given an arrow $\alpha \in Q_{1}$, the vertex $o(\alpha)(\operatorname{resp} t(\alpha))$ is called the origin (resp. terminus) of $\alpha$.

If $n$ is a natural number, then a path of length $n$ is just a vertex, when $n=0$, or a concatenation $p=\alpha_{1} \alpha_{2} \ldots \alpha_{n}$ of arrows, with $t\left(\alpha_{i}\right)=o\left(\alpha_{i+1}\right)$ for $i=1, \ldots, n-1$. In this later case $o(p):=o\left(\alpha_{1}\right)$ and $t(p):=t\left(\alpha_{n}\right)$ are called the origin and terminus of $p$, respectively. A vertex $i \in Q_{0}$ is considered to be a path of length 0 with origin and terminus equal to $i$. A walk or nonoriented path between two vertices $i$ and $j$ is a sequence $i=i_{0} \leftrightarrow i_{1} \leftrightarrow \ldots \leftrightarrow i_{r}=j$, where each edge $i_{k} \leftrightarrow i_{k+1}$ is eihter an arrow $i_{k} \rightarrow i_{k+1}$ or an arrow $i_{k} \leftarrow i_{k+1}$. The quiver is connected when, between any two vertices of $Q$, there is a walk.

The path $(R-)$ algebra of $Q$ is the free $R$ module $R Q$ with basis the set of paths in $Q$ on which one defines a multiplication of paths by the rule that $p \cdot q=0$, in case $t(p) \neq o(q)$, and $p \cdot q=p q$ is the concatenation of $p$ and $q$, provided that $t(p)=o(q)$. The multiplication on $R Q$ extends by $R$-linearity this multiplication of paths. If $i \in Q_{0}$ then one commonly uses the symbol $e_{i}$ to denote the vertex $i$, when viewed as an element of $R Q$. Note that $R Q$ is an algebra with enough idempotents, where $\left(e_{i}\right)_{i \in Q_{0}}$ is a distinguished family of nonzero orthogonal idempotents.

We denote by $R Q_{\geq n}$ the ideal of $R Q$ consisting of the $R$-linear combinations of paths of length $\geq n$. An admissible set of relations in $R Q$ is just a subset $\rho \subset \bigcup_{i, j \in Q_{0}} e_{i} R Q e_{j}$ such that each $r \in \rho$ is an $R$-linear combination of paths of length $\geq 2$. A (two-sided) ideal $I$ of $R Q$ is called admissible when it is generated by an admissible set of relations. An algebra $A$ with enough idempotents is said to be given by quivers and relations when there is a quiver $Q$ and an admissible set of relations $\rho$ such that $A=R Q / I$, where $I=\langle\rho\rangle$ is the ideal of $R Q$ generated by $\rho$. We still denote by $e_{i}$ its image by the projection $R Q \rightarrow R Q / I=A$. Then $\left(e_{i}\right)_{i \in I}$ is also a distinguished family of nonzero orthogonal idempotents of $A$ and a Wedderburn-Malcev type decomposition of $A$ is given by $A=B \oplus J$, where $B=\oplus_{i \in Q_{0}} R e_{i}$ and $J=R Q_{\geq 1} / I$. It will be the only one that we use in the rest of the paper and will be called the canonical Wedderburn-Malcev type decomposition of $A$.

We shall say that an algebra with enough idempotents is a locally finitely presented algebra when it is given by quiver and relations $(Q, \rho)$, and the set $\rho e_{i}=\rho \cap R Q e_{i}$ is finite, for all $i \in Q_{0}$.

The quiver $Q$ is called left (resp. right) locally finite when each vertex is the terminus (resp. origin) of, at most, a finite number of arrows. We say that $Q$ is locally finite when it is left and right locally finite. The set $Q_{0}$ admits a canonical preorder $\preceq$, where $i \preceq j$ if there is a path $p$ in $Q$ with $o(p)=i$ and $t(p)=j$. Note that $\preceq$ is an order relation exactly when $Q$ does not have oriented cycles. We shall say that $Q$ is a downward directed quiver (without minimum) when the preordered set $\left(Q_{0}, \preceq\right)$ is downward directed (without minimum). We will say that $Q$ is a narrow quiver if, given vertices $j \preceq i$, the set 


$$
\left\{k \in Q_{0}: k \preceq i \text { and } k \npreceq j\right\}
$$

is finite.

Recall (see [25) that if $\Delta$ is any quiver, then the associated translation quiver $\mathbb{Z} \Delta$ has $(\mathbb{Z} \Delta)_{0}=\mathbb{Z} \times \Delta_{0}$ as its set of vertices and, for each arrow $\alpha: o(\alpha) \longrightarrow t(\alpha)$ in $\Delta$, there are two arrows $(k, \alpha):(k, o(\alpha)) \rightarrow$ $(k, t(\alpha))$ and $\left(k, \alpha^{*}\right):(k, t(\alpha)) \longrightarrow(k+1, o(\alpha))$ in $\mathbb{Z} \Delta$.

Examples 5.3. $\quad$ 1. If $\Delta$ is a finite connected quiver, then $\mathbb{Z} \Delta$ is a connected quiver which is locally finite, downward directed and narrow.

2. If $\Delta=\mathbf{A}_{\propto}$ : $\quad 1 \rightarrow 2 \rightarrow \ldots \rightarrow n \rightarrow \ldots$, then $\mathbb{Z} \Delta$ is connected, locally finite and downward directed, but it is not narrow.

If $A$ is given by the quiver with relations $(Q, \rho)$, then, for every vertex $i \in Q_{0}$, each relation $r \in \rho e_{i}$ can be written in the form $\sum_{\alpha \in Q_{1}, t(\alpha)=i} x(r, \alpha) \alpha$, for uniquely determined elements $x(r, \alpha) \in R Q_{\geq 1} e_{o(\alpha)}$. Note that, since $\rho \subset \bigcup_{j, k \in Q_{0}} e_{j} R Q e_{k}$, there is a unique $j=j(r) \in Q_{0}$ such that $e_{j} r \neq 0$. Then we can assume that $x(r, \alpha)=e_{j} x(r, \alpha) e_{o(\alpha)}$, for each $\alpha \in Q_{1}$ such that $t(\alpha)=i$. We put $o(r)=j$. The following result is well-known when $R=K$ is a field. We give a proof in our more general setting.

Lemma 5.4. Let $A$ be given by a quiver with relations $(Q, \rho)$. For each $i \in Q_{0}$, there is a projective resolution of $B e_{i}=R e_{i}$ in $A-\operatorname{Mod}$

$$
\ldots \rightarrow \oplus_{r \in \rho e_{i}} A e_{o(r)} \stackrel{f}{\longrightarrow} \oplus_{\alpha \in Q_{1}, t(\alpha)=i} A e_{o(\alpha)} \stackrel{g}{\longrightarrow} A e_{i} \longrightarrow R e_{i} \rightarrow 0,
$$

where the maps $f$ and $g$ are determined by the equalities:

$$
f\left(e_{o(r)}\right)=\sum_{\alpha \in Q_{1}, t(\alpha)=i} x(r, \alpha) e_{o(\alpha)} \quad g\left(e_{o(\alpha)}\right)=\alpha,
$$

for each $r \in \rho e_{i}$ and each $\alpha \in Q_{1}$ with $t(\alpha)=i$.

Proof. We follow standard ideas which work for unital algebras over a field (see, e.g., 3]). The map $g^{\prime}: \oplus_{\alpha \in Q_{1}, t(\alpha)=i} R Q e_{o(\alpha)} \longrightarrow R Q_{\geq 1}$ mapping $\sum f_{\alpha} \rightsquigarrow \sum f_{\alpha} \alpha$ is an isomorphism of left $R Q$-modules, thus showing that the sequence

$$
0 \rightarrow \oplus_{\alpha \in Q_{1}, t(\alpha)=i} R Q e_{o(\alpha)} \stackrel{g^{\prime}}{\longrightarrow} R Q e_{i} \stackrel{p}{\longrightarrow} R e_{i} \rightarrow 0
$$

is a projective resolution of $R e_{i}$ as a left $R Q$-module. Applying the functor $A \otimes_{R Q}$ ? to this sequence, we obtain an exact sequence in $A-\operatorname{Mod}$

$$
0 \rightarrow \operatorname{Tor}_{1}^{R Q}\left(A, R e_{i}\right) \longrightarrow \oplus_{\alpha \in Q_{1}, t(\alpha)=i} A e_{o(\alpha)} \stackrel{g}{\longrightarrow} A e_{i} \stackrel{\bar{p}}{\longrightarrow} R e_{i} \rightarrow 0 .
$$

The functor $A \otimes_{R Q}$ ? leaves unaltered the exact sequence $0 \rightarrow \operatorname{Ker}(\bar{p}) \stackrel{i n c l}{\longrightarrow} A e_{i} \stackrel{\bar{p}}{\longrightarrow} R e_{i} \rightarrow 0$, which we view as a sequence of left $R Q$-modules annihilated by $I=\langle\rho\rangle$. The long exact sequence of Tor then gives an exact sequence in $A-\operatorname{Mod}$

$$
\operatorname{Tor}_{1}^{R Q}\left(A, A e_{i}\right) \stackrel{u}{\longrightarrow} \operatorname{Tor}_{1}^{R Q}\left(A, R e_{i}\right) \stackrel{0}{\longrightarrow} \operatorname{Ker}(\bar{p}) \stackrel{\text { incl }}{\longrightarrow} A e_{i},
$$

which shows that we have an epimorphism $\operatorname{Tor}_{1}^{R Q}\left(A, A e_{i}\right) \stackrel{u}{\rightarrow} \operatorname{Tor}_{1}^{R Q}\left(A, R e_{i}\right)$. Moreover, by a classical argument as in the case of unital algebras (see, e.g., [4, Chapter VIII, Section 1]), we have an isomorphism $\frac{I e_{i}}{I^{2} e_{i}} \stackrel{\cong}{\longrightarrow} \operatorname{Tor}_{1}^{R Q}\left(A, A e_{i}\right)$. We now consider the composition of morphisms in $A-\operatorname{Mod}$

$$
h: \frac{I e_{i}}{I^{2} e_{i}} \stackrel{\cong}{\longrightarrow} \operatorname{Tor}_{1}^{R Q}\left(A, A e_{i}\right) \stackrel{u}{\longrightarrow} \operatorname{Tor}_{1}^{R Q}\left(A, R e_{i}\right) \longrightarrow \oplus_{\alpha \in Q_{1}, t(\alpha)=i} A e_{o(\alpha)}=\oplus_{\alpha \in Q_{1}, t(\alpha)=i} \frac{R Q e_{o(\alpha)}}{I e_{o(\alpha)}} .
$$


It is easy to check that this map is induced by the restriction to $I e_{i}$ of the canonical map $\bar{h}: R Q e_{i} \longrightarrow$ $\oplus_{\alpha \in Q_{1}, t(\alpha)=i} R Q e_{o(\alpha)}$ which takes a path $p=q \alpha$ to $q$, where $\alpha$ is the last arrow of $p$.

If now $x \in I e_{i}$ and we write it as $x=\oplus_{\alpha \in Q_{1}, t(\alpha)=i} x(\alpha) \alpha$, for uniquely determined $x(\alpha) \in R Q e_{o(\alpha)}$, we have that $h\left(x+I^{2} e_{i}\right)=\sum_{\alpha \in Q_{1}, t(\alpha)=i}\left(x(\alpha)+I e_{o(\alpha)}\right)$. Then $h\left(x+I^{2} e_{i}\right)=0$ if, and only if, $x(\alpha) \in I e_{o(\alpha)}$ for each $\alpha \in Q_{1}$ with $t(\alpha)=i$. It follows that $\operatorname{Ker}(h)=\frac{I^{2} e_{i}+\left(\oplus_{\alpha \in Q_{1}, t(\alpha)=i} I \alpha\right)}{I^{2} e_{i}}=\frac{I^{2} e_{i}+I \cdot R Q_{>1} e_{i}}{I^{2} e_{i}}$.

The above paragraphs show that we have an exact sequence in $A-\operatorname{Mod}$

$$
0 \rightarrow \frac{I^{2} e_{i}+I \cdot R Q_{\geq 1} e_{i}}{I^{2} e_{i}} \hookrightarrow \frac{I e_{i}}{I^{2} e_{i}} \stackrel{h}{\longrightarrow} \oplus_{\alpha \in Q_{1}, t(\alpha)=i} A e_{o(\alpha)} \stackrel{g}{\longrightarrow} A e_{i} \stackrel{\bar{p}}{\longrightarrow} R e_{i} \rightarrow 0 .
$$

This shows that we have an isomorphism $\tilde{h}: \frac{I e_{i}}{I^{2} e_{i}+I \cdot R Q_{\geq 1} e_{i}} \stackrel{\cong}{\cong} \operatorname{Ker}(g)$ which maps $\sum_{\alpha \in Q_{1}, t(\alpha)=i} x(\alpha) \alpha+$ $\left(I^{2} e_{i}+I \cdot R Q_{\geq 1}\right)$ onto $\sum_{\alpha \in Q_{1}, t(\alpha)=i} x(\alpha) e_{o(\alpha)}$. We just need to check that $\frac{I e_{i}}{I^{2} e_{i}+I \cdot R Q_{\geq 1}}$ is generated, as a left $A$-module, by the elements $\bar{r}=r+\left(I^{2} e_{i}+I \cdot R Q_{\geq 1}\right)$, where $r$ varies in the set $\rho e_{i}$. Indeed, if this is the case then we will have a canonical epimorphism of left $A$-modules $\oplus_{r \in \rho e_{i}} A e_{o(r)} \rightarrow \frac{I e_{i}}{I^{2} e_{i}+I \cdot R Q_{\geq 1} e_{i}}$ mapping $e_{o(r)} \rightarrow \bar{r}$, for each $r \in \rho e_{i}$. The composition of this epimorphism followed by $\tilde{h}$ and by the inclusion $\operatorname{Ker}(g) \rightarrow \oplus_{\alpha \in Q_{1}, t(\alpha)=i} A e_{o(\alpha)}$ is just the map $f$ in the statement and the proof will be finished.

To check what is needed, take $x \in I e_{i}$. Then we can express it in the form $x=\sum_{1 \leq j \leq m} \lambda_{j} p_{j} r_{j} q_{j}$, where $0 \neq \lambda_{j} \in R, r_{j} \in \rho$ is a relation and $p_{j}$ and $q_{j}$ are paths with $t\left(p_{j}\right)=o\left(r_{j}\right)$ and $o\left(q_{j}\right)=t\left(r_{j}\right)$, $t\left(q_{j}\right)=i$. Note that if length $\left(q_{j}\right)>0$ then $p_{j} r_{j} q_{j}$ is in $I \cdot R Q_{\geq 1} e_{i}$ and, hence, it becomes zero in $\frac{I e_{i}}{I^{2} e_{i}+I \cdot R Q_{\geq 1} e_{i}}$. Therefore we can assume that length $\left(q_{j}\right)=0$ (i.e. $q_{j}=e_{i}$ ), for all $j=1, \ldots, m$. But then all the $r_{j}$ are in $\rho e_{i}$ and we can rewrite $x$ as $\sum_{r \in \rho e_{i}} a(r) r$, for some elements $a(r) \in R Q e_{o(r)}$. Then $\frac{I e_{i}}{I^{2} e_{i}+I \cdot R Q_{\geq 1}}$ is generated by the $\bar{r}$ as a left $A$-module, as desired.

Proposition 5.5. Let $Q$ be a quiver which is connected, left locally finite, downward directed, narrow and has no oriented cycles, and let $A$ be an algebra locally finite presented by the quiver with relations $(Q, \rho)$. Then the following assertions hold:

1. $J e_{i}$ is finitely presented and $J^{m} e_{i}$ is finitely generated, as left ideals, for all $i \in Q_{0}$ and all $m>0$

2. The order relation $\preceq$ is the smallest preorder relation in $Q_{0}$ containing all the pairs $(i, j)$ such that $e_{i} A e_{j} \neq 0$

3. For all vertices $j \preceq i$, the left A-module $\frac{A e_{i}}{\sum_{k \preceq j} A e_{k} A e_{i}}$ is annihilated by some $J^{n}$, with $n>0$

Proof. A path $p$ will be called a 'nonzero path' when its image by the projection $R Q \longrightarrow R Q / I=A$ is nonzero, where $I=\langle\rho\rangle$.

1) $J^{m} e_{i}$ is generated, as a left ideal, by all nonzero paths of length $m$ with terminus $i \in Q_{0}$. The left locally finite condition of $Q$ guarantees that there are only a finite number of such paths.

With the notation of the preceding lemma, we have $\operatorname{Im}(g)=\operatorname{Ker}(p)=J e_{i}$. The fact that $J e_{i}$ is finitely presented as a left ideal follows from lemma 5.4 and the finiteness of $\rho e_{i}$.

$2) \preceq$ is the smallest preorder relation in $Q_{0}$ containing all the pairs $(i, j) \in Q_{0} \times Q_{0}$ such that there is an arrow $i \rightarrow j$ in $Q$. For such a pair it always happens that $e_{i} A e_{j} \neq 0$ because a linear combination of arrows is never in $I=\langle\rho\rangle$. Conversely, if $e_{i} A e_{j} \neq 0$ then there is a nonzero path $p$ such that $o(p)=i$ and $t(p)=j$, which implies that $i \preceq j$.

$3)$ Let $j \prec i$ be any vertices. By the narrow condition of $Q$, there are $k_{1}, \ldots, k_{r} \in Q_{0}$ such that

$$
\left\{k \in Q_{0}: k \preceq i \text { and } k \npreceq j\right\}=\left\{k_{1}, \ldots, k_{r}\right\} .
$$

Now the left locally finite condition of $Q$ and the fact that $Q$ has no oriented cycles imply that the paths with origin a $k_{t}$ and terminus $i$ have a maximal length $n$. Then any path $p$ in $Q$ with $t(p)=i$ and length $m>n$ has the property that $o(p) \preceq j$. Then we have $p \in \sum_{k \preceq j} A e_{k} A e_{i}$, and hence $J^{m} e_{i} \subseteq$ $\sum_{k \preceq j} A e_{k} A e_{i}$. 
Recall that a hereditary torsion class $\mathcal{T}^{\prime}$ in a Grothendieck category $\mathcal{G}$ is itself a Grothendieck category. Indeed, it is clearly abelian, with kernels and cokernels of morphisms calculated as in $\mathcal{G}$, and it is cocomplete with coproducts also calculated as in $\mathcal{G}$. Moreover, the inclusion functor $j: \mathcal{T}^{\prime} \hookrightarrow \mathcal{G}$ preserves colimits, since it is a left adjoint functor, and it is exact. Then direct limits in $\mathcal{T}^{\prime}$ are exact because they are so in $\mathcal{G}$. Finally, if $G$ is a generator of $\mathcal{G}$, then the epimorphic images of $G$ which are in $\mathcal{T}^{\prime}$ form a set of generators of $\mathcal{T}^{\prime}$.

We are now ready to prove the following result, where the grading considered in $R Q$ is the length grading, i.e., the one for which each path is homogeneous of degree equal to its length.

Theorem 5.6. Let $A$ be an algebra with enough idempotents locally finitely presented by the quiver with relations $(Q, \rho)$, where $Q$ is connected, locally finite and has no oriented cycles. Let $A=B \oplus J$ its canonical Wedderburn-Malcev type decomposition and let $\mathcal{T}$ be the class of torsion A-modules, whose torsion radical is denoted by $t$. The following assertions hold:

1. If $\rho$ consists of homogeneous relations, $t(A)=0$ and, for each $i \in Q_{0}$, there are only finitely many vertices $j$ such that $i \preceq j$, then $\mathcal{T}$ is a locally finitely presented Grothendieck category with no nonzero flat objects.

2. If $Q$ is downward directed and narrow and $\operatorname{Ext}_{A}^{1}\left(B e_{i}, \frac{A e_{j}}{t(A) e_{j}}\right)=0$, for all $i, j \in Q_{0}$, then $\mathcal{G} / \mathcal{T}$ is a locally finitely presented Grothendieck category with no nonzero flat objects.

Proof. 1) By the proof of the preceding proposition, we know that $J e_{i}$ is finitely presented and $J^{m} e_{i}$ is finitely generated, as left ideals, for all $i \in Q_{0}$ and all $m>0$. Then, by lemma 5.1, we know that $\mathcal{T}$ is a hereditary torsion class of finite type in $A-$ Mod. Moreover $\mathcal{S}:=\left\{\frac{A e_{i}}{J^{m} e_{i}}: i \in Q_{0}, m>0\right\}$ is a set of finitely presented generators of $\mathcal{T}$. On the other, hand each finitely generated $A$-module $T$ in $\mathcal{T}$ is annihilated by some power $J^{m}$ and, hence, it is canonically a $A / J^{m}$-module. It follows that it is a direct limit of finitely presented $A / J^{m}$-modules, all of which are also finitely presented objects of $\mathcal{T}$. The fact that each object of $\mathcal{T}$ is a direct union of its finitely generated submodules implies, by suitable arrangement of direct systems, that each object of $\mathcal{T}$ is a direct limit of finitely presented objects of $\mathcal{T}$. Therefore $\mathcal{T}$ is a locally finitely presented Grothendieck category.

Note that, since we have epimorphisms $\frac{A e_{i}}{J^{n+1} e_{i}} \rightarrow \frac{A e_{i}}{J^{n} e_{i}}$ for all $i \in Q_{0}$ and $n>0$, it follows that the set $\mathcal{S}(m):=\left\{\frac{A e_{i}}{J^{m} e_{i}}: i \in Q_{0}\right\}$ is a set of generators of $\mathcal{T}$, for each integer $m>0$. Suppose now that there exists a flat object $F \neq 0$ in $\mathcal{T}$. Let us fix any nonzero morphism $f: A e_{i} \longrightarrow F$ in $A-$ Mod, which exists for some $i \in Q_{0}$. Then we can fix an integer $k>0$ such that $J^{k} e_{i} \subseteq \operatorname{Ker}(f)$ because $\operatorname{Im}(f)$ is cyclic and all cyclic modules in $\mathcal{T}$ are annihilated by some power of $J$. We then consider the induced morphism $\bar{f}: \frac{A e_{i}}{J^{k} e_{i}} \longrightarrow F$. By hypothesis, there are only finitely many vertices $j \in Q_{0}$ such that $i \preceq j$. This together with the locally finite condition of $Q$ imply that there are only finitely many paths in $Q$ with origin $i$. Let $t$ be the maximal length of these paths. We now fix any integer $m>k+t$ and consider an epimorphism $p: \oplus_{j \in Q_{0}} \frac{A e_{j} J^{m} e_{j}}{\left(H_{j}\right)} \rightarrow F$. Due to the flatness of $F$, this epimorphism is pure and, hence, $\bar{f}$ should factor through $p$. But this is impossible since $\operatorname{Hom}_{A}\left(\frac{A e_{i}}{J^{k} e_{i}}, \frac{A e_{j}}{J^{m} e_{j}}\right)=0$, for all $j \in Q_{0}$. Indeed, the existence of a nonzero morphism $g: \frac{A e_{i}}{J^{k} e_{i}} \longrightarrow \frac{A e_{j}}{J^{m} e_{j}}$ implies the existence of a morphism $\frac{A e_{i}}{J^{k} e_{i}} \longrightarrow \frac{A e_{j}}{J^{m} e_{j}}[r]$ in $A-G r$, for a suitable integer $r$. Then we can assume that $g$ is a graded morphism of some degree. It is then determined by an element $0 \neq x \in e_{i} A e_{j}$ which is an $R$-linear combination of paths $i \rightarrow \ldots \rightarrow j$ of the same length, say $u$, such that $p x \in J^{m} e_{j}$, for all paths $p$ of length $k$ with $t(p)=i$. Then we have $i \preceq j$ and, hence, necessarily $u \leq t$. But if $p x \neq 0$, then $\operatorname{deg}(p x)=\operatorname{deg}(p)+\operatorname{deg}(x)=k+u \leq k+t<m$. This is impossible since $p x \in J^{m} e_{j}$ and all nonzero homogeneous elements of $J^{m} e_{j}$ have degree $\geq m$. It follows that $p x=0$ in $A$, for all paths $p$ of length $k$ with $t(p)=i$, or, equivalently, that $J^{k} x=0$. The fact that $t(A)=0$ implies that this can only happen when $x=0$, which is a contradiction.

2) If $\operatorname{Ext}_{A}^{1}\left(B e_{i}, \frac{A e_{j}}{t(A) e_{j}}\right)=0$, for all $i, j \in Q_{0}$, then, for any integer $m>0$, one easily shows by reverse induction that $\operatorname{Ext}_{A}^{1}\left(\frac{J^{k} e_{i}}{J^{m} e_{i}}, \frac{A e_{j}}{t(A) e_{j}}\right)=0$ for $k=m, m-1, \ldots, 0$. It follows that $\operatorname{Ext}_{A}^{1}\left(\frac{A e_{i}}{J^{m} e_{i}}, \frac{A e_{j}}{t(A) e_{j}}\right)=0$, for 
all $i, j \in Q_{0}$. On the other hand, if $\operatorname{Hom}_{A}\left(A e_{i}, \frac{A e_{j}}{t(A) e_{j}}\right) \neq 0$ then $e_{i} A e_{j} \cong \operatorname{Hom}_{A}\left(A e_{i}, A e_{j}\right)$ is nonzero, due to the projective condition of $A e_{i}$. This implies that $i \preceq j$. Then the set of indices $j \prec i$ for which either $\operatorname{Hom}_{A}\left(A e_{i}, \frac{A e_{j}}{t(A) e_{j}}\right) \neq 0$ or $\operatorname{Ext}_{A}^{1}\left(\frac{A e_{i}}{J^{m} e_{i}}, \frac{A e_{j}}{t(A) e_{j}}\right) \neq 0$, for some integer $m>0$, is the empty set. It follows that condition 2 in proposition 5.2 holds. Then, by using proposition [5.5, we see that all the hypotheses of proposition 5.2 hold. Therefore $A-\operatorname{Mod} / \mathcal{T}$ is a locally finitely presented Grothendieck category without nonzero flat objects.

If $Q$ is a quiver and $K$ is a field, then the path algebra $K Q$ has a canonical structure of coalgebra, which we denote $K^{\square} Q$, where the counit $\epsilon: K^{\square} Q \longrightarrow K$ is the $K$-linear map which vanishes on all paths of positive length and takes $e_{i} \rightsquigarrow 1$, for all $i \in Q_{0}$, and the comultiplication $\Delta: K^{\square} Q \longrightarrow K^{\square} Q \otimes K^{\square} Q$ maps $e_{i} \rightsquigarrow e_{i} \otimes e_{i}$, for each $i \in Q_{0}$, and

$$
\Delta(p)=e_{o(p)} \otimes p+\sum_{1 \leq s \leq m-1} \beta_{1} \ldots \beta_{s} \otimes \beta_{s+1} \ldots \beta_{m}+p \otimes e_{t(p)},
$$

for each path of positive length $p=\beta_{1} \cdot \ldots \cdot \beta_{m}$.

If $K$ is a field, $(Q, \rho)$ is a quiver with relations over $K$ is a field and $I=\langle\rho\rangle$ is the ideal of $K Q$ generated by $\rho$, then the associated $\left(K\right.$-)coalgebra $C(Q, \rho)$ is the subcoalgebra $I^{\perp}$ of $K^{\square} Q$, where $I^{\perp}$ denotes the right orthogonal of $I$ with respect to the nondegenerate bilinear form $(-,-): K Q \times$ $K^{\square} Q \longrightarrow K$ which maps a pair $(p, q)$ of paths to the Kronecker element $\delta_{p q}$. Obviously, if $\rho$ consists of homogeneous elements, then $I$ is a graded ideal and the coalgebra $C(Q, \rho)$ is a graded coalgebra (i.e. $\left.\Delta\left(C_{n}\right) \subseteq \sum_{r+s=n} C_{r} \otimes C_{s}\right)$. Example 2.11 in [8] gives a path coalgebra whose category of comodules has no nonzero flat objects. Our next result shows that this is not an exceptional fact for coalgebras. The reader is referred to [29] for the terminology and results that we use.

Corollary 5.7. Let $C(Q, \rho)$ be the $K$-coalgebra associated to the quiver with relations $(Q, \rho)$. Suppose that the following conditions hold:

1. $Q$ is connected, locally finite and has no oriented cycles. Moreover, for each $i \in Q_{0}$, there are only finitely many $j \in Q_{0}$ such that $i \preceq j$

2. $\rho$ consists of homogeneous elements with respect to the length grading on $K Q$ and, for each $i \in Q_{0}$, the set $\rho e_{i}$ is finite.

3. The algebra $A=K Q /\langle\rho\rangle$ has no nonzero element $x$ such that $A_{1} x=0$ (equivalently, $C$ ) contains maximal right subcomodule)

Then the category Comod $-C$ of right $C$-comodules contains no nonzero flat objects.

Proof. Note that if $A=K Q /\langle\rho\rangle$, then the category of left $A$-modules is equivalent to the category $\operatorname{Rep}_{K}\left(Q^{o p}, \rho^{o p}\right)$ of $K$-representations of the opposite quiver with relations $\left(Q^{o p}, \rho^{o p}\right)$. Under this equivalence, due to the properties of our quiver, the class $\mathcal{T}$ of torsion $A$-modules corresponds to the class $\operatorname{Rep}_{K}^{\text {lnlf }}\left(Q^{o p}, \rho^{o p}\right)$ of locally finite nilpotent representations of $\left(Q^{o p}, \rho^{o p}\right)$ in the terminology of [29]. Clearly $C\left(Q^{o p}, \rho^{o p}\right)$ is the opposite coalgebra $C:=C(Q, \rho)$, i.e., if we use the classical $\Sigma$-notation of Sweedler [32] and $\Delta: C \longrightarrow C \otimes C$ is the comultiplication of $C$, then $\Delta^{o}: C^{o p} \longrightarrow C^{o p} \otimes C^{o p}$ is given by $\Delta^{o}\left(c^{o}\right)=\sum c_{2}^{o} \otimes c_{1}^{o}$, provided $\Delta(c)=\sum c_{1} \otimes c_{2}$, and viceversa.

Then, using [29, Theorem 4.5], we have equivalences of categories

$$
\mathcal{T} \stackrel{\cong}{\longleftrightarrow} \operatorname{Rep}_{K}^{\text {lnlf }}\left(Q^{o p}, \rho^{o p}\right) \stackrel{\cong}{\longleftrightarrow} C^{o p}-\operatorname{Comod} \stackrel{\cong}{\leftrightarrows} \operatorname{Comod}-C,
$$

and the result follows from theorem 5.6 .

We finally give an example, derived from corollary 4.5, where assertion 2 of theorem 5.6 applies. To any $n+1$ variables $x_{0}, \ldots, x_{n}$, we associate the quiver 


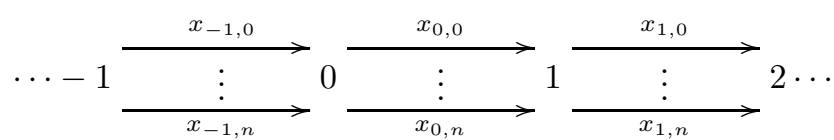

That is, we have $Q_{0}=\mathbb{Z}$ and, for each $k \in \mathbb{Z}$, we have $n+1$ arrows $x_{k, j}: k \rightarrow k+1$, where $j=0,1, \ldots, n$. For each $k \in \mathbb{Z}$ and each (noncommutative) monomial $\mu=x_{j_{1}} \ldots x_{j_{d}}$ of degree $d$ in the $x_{j}$, we have a path $\mu_{k}: k \rightarrow k+1 \rightarrow \ldots \rightarrow k+d$ of length $d$ given by $\mu_{k}: x_{k, j_{1}} \ldots x_{k+d-1, j_{d}}$. If now $h \in R<x_{0}, x_{1}, \ldots, x_{n}>$ is any homogeneous element of degree $d$ in the free $R$-algebra on the $x_{j}$, written as $h=\sum \lambda_{\mu} \mu$, where the sum ranges over the set of monomials $\mu$ of degree $d$ and $\lambda_{\mu} \in R$, then we put $h_{k}:=\sum \lambda_{\mu} \mu_{k}$. This is a well-defined element of $R Q$.

Corollary 5.8. Let $R$ be a commutative Noetherian domain, let $\mathbf{p}$ be a prime homogeneous ideal of $A=R\left[x_{0}, x_{1}, \ldots, x_{n}\right]$ contained in $\left(x_{0}, x_{1}, \ldots, x_{n}\right)^{2}$ and let $I_{\mathbf{p}}$ be the homogeneous ideal of RQ generated by all the congruencies $x_{k, i} x_{k+1, j}-x_{k, j} x_{k+1, i}$ and all the $h_{k}$, where $k \in \mathbb{Z}$ and $h$ is a homogeneous polynomial in $\mathbf{p}$. Put $\tilde{A}=R Q / I_{\mathbf{p}}$ and let $\mathcal{T}$ be the category of torsion left $\tilde{A}$-modules.

If the height of $\left(x_{0}, \ldots, x_{n}\right) / \mathbf{p}$ in $A$ is $\geq 2$ and $A$ is either integrally closed or Cohen-Macaulay, then $\tilde{A}-\operatorname{Mod} / \mathcal{T}$ is a locally finitely presented Grothendieck category with no nonzero flat objects.

Proof. By the proof of corollary 4.5, we know that $\operatorname{Ext}_{A}^{1}(R, A)=0$. We look now at $A$ as a factor of the path algebra of the quiver $Q^{\prime}$ which has one vertex and $n+1$ loops at it, i.e., as a factor of the free (noncommutative) $R$-algebra on $n+1$ variables. Then $A=R Q^{\prime} / I$, where $I$ is generated by a finite set $\rho$ of homogeneous elements of $R Q^{\prime}$, consisting of the commutativity relations $x_{i} x_{j}-x_{j} x_{i}(i, j=0,1, \ldots, n)$ and a finite set of homogeneous generators of $\mathbf{p}$, which are viewed as elements of $R<x_{0}, x_{1}, \ldots, x_{n}>$. It is clear that $\hat{\rho}=\left\{h_{k}: h \in \rho, k \in \mathbb{Z}\right\}$ generates $I_{\mathbf{p}}$ as an ideal of $R Q$. Moreover, there is a bijection between $\rho$ and $\hat{\rho} e_{k}$, thus showing that $\hat{\rho} e_{k}$ is finite, for each $k \in \mathbb{Z}$.

We now use the theory of Galois coverings of quivers with relations (see [1] and [19], for the old theory, and [5] and [2] for the most recent one, specially the last paper, where the theory is developed over an arbitrary commutative ring as we need). We know that $(Q, \hat{\rho})$ is a Galois covering of $\left(Q^{\prime}, \rho\right)$. That gives a push-down functor $F: \tilde{A}-\operatorname{Mod} \longrightarrow A-\operatorname{Mod}$ which takes torsion $\tilde{A}$-modules to $A$-modules whose elements are annihilated by powers of $\left(x_{0}, x_{1}, \ldots, x_{n}\right)$. It also takes a projective resolution of $R e_{k}$ to a (graded) projective resolution of $R$ as an $A$-module. If

$$
\rightarrow P^{-2} \stackrel{d^{-2}}{\longrightarrow} P^{-1} \stackrel{d^{-1}}{\longrightarrow} P^{0} \rightarrow R e_{k} \rightarrow 0
$$

is the initial part of a projective resolution as given in lemma 5.4 (with $\tilde{A}$ instead of $A$ ), then we view it as a graded one, by putting $P^{0}=\tilde{A} e_{k}[0], P^{-1}=\tilde{A} e_{k-1}[-1]^{(n+1)}$ and $P^{-2}=\oplus_{r \in \rho} \tilde{A} e_{k-d(r)}[-d(r)]$, where $d(r)$ is the degree of $r$, for each $r \in \rho$. Then the functor $F$ takes it to the following initial part of a graded projective resolution of $R$ as an $A$-module:

$$
\oplus_{r \in \rho} A[-d(r)] \longrightarrow A[-1] \longrightarrow A \longrightarrow R \rightarrow 0
$$

If $f: P^{-1} \longrightarrow \tilde{A} e_{j}$ is a graded morphism of $\tilde{A}$-modules, say of degree $t$, such that $f \circ d^{-2}=0$, then $F(f) \circ F\left(d^{-2}\right)=0$ and the fact that $\operatorname{Ext}_{A}^{1}(R, A)=0$, and hence $\operatorname{Ext}_{A-G r}^{1}(R, A[t])=0$, gives a graded morphism of $A$-modules $g: F\left(P^{0}\right)=R \longrightarrow F\left(A e_{j}\right)=R$ such that $g \circ F\left(d^{-1}\right)=F(f)$.

By the properties of the push-down functor, we have a graded morphism $\hat{g}: P^{0}=\tilde{A} e_{k} \longrightarrow \tilde{A} e_{j}$ of the same degree that $g$ such that $F(\hat{g})=g$ and $\hat{g} \circ d^{-1}=f$. It follows that $\operatorname{Ext}_{\tilde{A}-G r}^{1}\left(R e_{k}, \tilde{A} e_{j}[t]\right)=0$, for all $j, k \in Q_{0}$ and $t \in \mathbb{Z}$ and, hence, also $\operatorname{Ext}_{\tilde{A}}^{1}\left(B e_{k}, \tilde{A} e_{j}\right)=\operatorname{Ext}_{\tilde{A}}^{1}\left(R e_{k}, \tilde{A} e_{j}\right)=0$, where $B=\oplus_{i \in Q_{0}} R e_{i}(\operatorname{see}$ the beginning of section 3 ).

Bearing in mind proposition [5.5, we conclude that all hypotheses of assertion 2 in theorem 5.6 are satisfied by $\tilde{A}$ and, hence, $\tilde{A}-\operatorname{Mod} / \mathcal{T}$ is a locally finitely presented Grothendieck category with no nonzero flat objects. 


\section{References}

[1] J. AdÁmek And J. Rosický, Locally presentable and accessible categories. London Math. Soc Lect. Notes Ser. 189. Cambridge Univ. Press 1994.

[2] H. Asashiba, A generalization of Gabriel's Galois covering functors and derived equivalences. J. Algebra 334 (2011), 109-149.

[3] M.C.R. Butler And A.D. KIng, Minimal resolutions of algebras. J. Algebra 212 (1999), 323-362.

[4] H. Cartan and S. Eilenberg, Homological Algebra, 7th edition. Princeton Univ. Press 1973.

[5] C. Cibils and E.N. Marcos, Skew category, Galois covering and smash product of a k-category. Proc. Amer. Math. Soc. 134(1) (2006), 39-50.

[6] W. Crawley-Boevey, Locally finitely presented additive categories. Comm. Algebra 22(1994), $1641-1674$

[7] S. Crivei, M. Prest and B. Torrecillas, Covers in finitely accessible categories. Proc. Amer. Math. Soc 138 (2010), 1213-1221.

[8] J. Cuadra and D. Simson, Flat comodules and perfect coalgebras. Comm. Algebra 35 (2007), 3164-3194.

[9] E.E. Enochs And S. Estrada, Relative homological algebra in the category of quasi-coherent sheaves. Adv. in Math. 194 (2005), 284-295.

[10] P. Gabriel, Des catégories abéliennes. Bull. Soc. Math. France. 90(1962), 323-448.

[11] P. Gabriel, The universal cover of a representation-finite algebra. Springer LNM 903 (1981), 68105.

[12] V. E. Govorov, On flat modules (in Russian). Sibirsk. Mat. Ž. 6 (1965), 300-304.

[13] A. Grothendieck, And J. A. Dieudonné, Eléments de géométrie algébrique I. Grundlehren math. Wiss, 166. Berlin-Heidelberg-New York, Springer-Verlag, 1971.

[14] R. Hartshorne, Algebraic Geometry, 6th edition, 1993.

[15] I. Herzog, Pure-injective envelopes. J. Algebra Appl. 2 (2003), 397-402.

[16] H. Krause, The spectrum of a module category, Mem. Amer. Math. Soc., 149 (2001), no. 707.

[17] E. Kunz, Introduction to commutative algebra and algebraic geometry. Birkhäuser Boston, Inc., Boston, MA, 1985.

[18] D. LazArD, Autour de la platitude. Bull. Soc. Math. France 97 (1969), 81-128.

[19] R. Martínez Villa AND J. A. DE LA PeÑA, The universal cover of a quiver with relations, J. Pure Appl. Algebra 30 (1983), 277-292.

[20] H. Matsumura, Commutative algebra, Second edition. Mathematics Lecture Note Series, 56. Benjamin/Cummings Publishing Co., Inc., Reading, Mass., 1980.

[21] D. Murfet, An adjunction for modules over projective schemes, available at www.therisingsea.org

[22] D. Murfet, Modules over projective schemes, available at www.therisingsea.org 
[23] C. Năstăsescu, and F. Van Oystaeyen, Graded and filtered rings and modules, Lecture Notes in Math., 758, Springer-Verlag, Berlin, 1979.

[24] M. Prest, AND A. RAlph, Locally finitely presented categories of sheaves of modules, available at http://www.maths.manchester.ac.uk/ mprest/publications.html

[25] C. Riedtmann, Algebren, Darstellungen, Überlagerungen und zurück, Comment. Math. Helv. 55 (1980). 199-224

[26] J.J. Rotman, An introduction to Homological Algebra. Academic Press 1979.

[27] W. Rump, Locally finitely presented categories of sheaves. J. Pure Appl. Algebra 214 (2010), 177186.

[28] W. Rump, Flat covers in abelian and in non-abelian categories. Adv. Math. 225 (2010), 1589-1615.

[29] D. Simson, Path coalgebras of profinite bound quivers, cotensor coalgebras of bound species and locally nilpotent representations. Colloq. Math. 109(2) (2007), 307-343.

[30] B. Stenström, Purity in functor categories. J. Algebra. 8 (1968), 352-361.

[31] B. Stenström, Rings of Quotients, GMW 217, Springer-Verlag, New York-Heidelberg 1975.

[32] M.E. Sweedler, Hopf algebras, W. A. Benjamin (1969). 